\title{
Stochastic linear-quadratic optimal control without time-consistency requirement
}

\author{
YuAN-HuA Ni AND Ji-FENG ZHANG
}

\begin{abstract}
In this paper, linear-quadratic optimal control without timeconsistency requirement is studied for a class of linear discretetime systems with multiplicative stochastic disturbances. Both the open-loop and the closed-loop time-consistent solutions are investigated. Necessary and sufficient conditions on the existence of the open-loop time-consistent equilibrium control and the closed-loop time-consistent equilibrium strategy are obtained, respectively. Specifically, the existence of the open-loop time-consistent equilibrium control for all the initial time-state pairs is equivalent to the solvability of two coupled constrained linear difference equations and two coupled constrained generalized difference Riccati equations; the existence of the closed-loop time-consistent equilibrium strategy is equivalent to the solvability of another two coupled constrained generalized difference Riccati equations. It can be found that Riccati equations for the open-loop formulation do not admit symmetry structure, while the ones for the closed-loop formulation do have symmetric solutions.
\end{abstract}

\section{Introduction}

By the Bellman optimality principle, we know that an optimal control for an initial pair will stay optimal thereafter. This property is also referred as the time-consistency of an optimal control, according to which one needs only to solve an optimal control problem for a given initial pair and the obtained optimal control is also optimal along the optimal trajectory. So, the timeconsistency provides a theoretical foundation of the dynamic programming

Yuan-Hua $\mathrm{Ni}$ is supported by the National Natural Science Foundation of China (11471242), the China Postdoctoral Science Foundation (2014M560128, 2015T80143). Ji-Feng Zhang is supported by the National Natural Science Foundation of China (61227902) and the National Key Basic Research Program of China (973 Program) under grant 2014CB845301. 
approach. In this paper, a class of stochastic linear-quadratic (LQ) optimal control problem is considered. Different from our recent works about meanfield LQ problems [8][9], a new feature of the considered problem is that the conditional expectations of the state and control appear quadratically in the cost functional. In this case, the smoothing property of conditional expectation cannot ensure the time-consistency of the optimal control. A particular example of this case is the well-known mean-variance utility [2] $[3]$.

We may ask: How to deal with the time-inconsistent optimal control problems? Let us take a look. Roughly speaking, finding an optimal control is a dynamical optimization problem, as time variable is involved. For a time-inconsistent optimal control problem, we could find an optimal control for an initial pair, and this control is optimal only viewed at the initial time or in a static sense. Alternatively, it is more reasonable for a decision maker to commit to his policy in an infinitesimal manner. In this case, some kinds of time-consistent equilibrium solutions are concerned with. This is mainly motivated by practical applications such as in mathematical finance and economics, and has attracted considerable interest and efforts. Recently, [4] and [5] studied the non-exponential discounting problems both for simple ordinary differential equations and stochastic differential equations, and introduced the notion of time-consistent control, while [3] started with the problems of general Markovian time-inconsistent stochastic optimal control. Concerned with the deterministic continuous-time LQ problems, [11] and [12] addressed it by an essentially cooperative game approach. This line is followed by a part of [14] to consider the stochastic LQ problem of meanfield type, which is called the closed-loop formulation there. Different from [11] [12], [6] studied another kind of time-consistent equilibrium control, which is an infinitesimally open-loop optimal control. As an application, a mean-variance portfolio selection problem is considered, and an equilibrium control is explicitly obtained. In [14], the author investigated both the open-loop and the closed-loop time-consistent solutions for general meanfield stochastic LQ problems; it is shown that the existence of the open-loop equilibrium control and the closed-loop equilibrium strategy is ensured via the solvability of certain Riccati-type equations.

In this paper, both the open-loop and the closed-loop time-consistent solutions are investigated for a general time-inconsistent discrete-time meanfield stochastic LQ problem. Here, we do not pose the definite constraint on the state and the control weighting matrices. Roughly speaking, the open-loop time-consistent solution is concerned with the time-consistency of an open-loop control, while the closed-loop solution is focusing on the 
time-consistency of a closed-loop strategy. In Section 2, the open-loop timeconsistent equilibrium control of Problem (LQ) is introduced. It is shown that the existence of an open-loop equilibrium control for an initial pair is equivalent to the solvability of a set of forward-backward stochastic difference equations (FBS $\Delta \mathrm{Es}$ ) with stationary condition and convexity condition. Moreover, the existence of the open-loop equilibrium control for all the initial pairs is shown to be equivalent to the solvability of two coupled linear difference equations (LDEs) and two coupled generalized difference Riccati equations (GDREs). In Section 3, the closed-loop time-consistent equilibrium strategy is investigated, whose existence is equivalent to the solvability of another two coupled GDREs. It is worth noticing that the GDREs for the open-loop formulation do not have symmetric structure, i.e., their solutions are not symmetric, although the ones for the closed-loop formulation have symmetric structure.

A static mean-field stochastic LQ optimal control problem is investigated in [9], where the expectations of the state and control appear quadratically in the cost functional. Deeply investigating the results of [9], we can see that the static mean-field LQ problem is indeed time-consistent. This is essentially different from what we are considering here. Furthermore, a time-inconsistent stochastic LQ problem is studied in [7], where the system equation and the cost functional are depending explicitly on the initial time.

The rest of this paper is organized as follows. Section 2 and Section 3 are considering the open-loop and closed-loop formulations, respectively. In Section 4, an example is presented and the paper ends with the concluding remarks in Section 5.

\section{Open-loop time-consistent equilibrium control}

Let us consider the following stochastic linear system with multiplicative disturbances

$$
\left\{\begin{array}{l}
X_{k+1}^{t}=\left(A_{k} X_{k}^{t}+B_{k} u_{k}\right)+\left(C_{k} X_{k}^{t}+D_{k} u_{k}\right) w_{k} \\
X_{t}^{t}=x, \quad k \in\{t, \ldots, N-1\} \triangleq \mathbb{T}_{t}, \quad t \in\{0,1, \ldots, N-1\} \triangleq \mathbb{T}
\end{array}\right.
$$

where $A_{k}, C_{k}, \in \mathbb{R}^{n \times n}$ and $B_{k}, D_{k} \in \mathbb{R}^{n \times m}$ are deterministic matrices; $\left\{X_{k}^{t}, k \in \widetilde{\mathbb{T}}_{t}\right\} \triangleq X^{t}$ with $\widetilde{\mathbb{T}}_{t}=\{t, t+1, \ldots, N\}$, and $\left\{u_{k}, k \in \mathbb{T}_{t}\right\} \triangleq u$ are the 
state process and the control process, respectively. The cost functional associated with system (1) is

$\begin{aligned} J(t, x ; u)= & \sum_{k=t}^{N-1} \mathbb{E}_{t}\left[\left(X_{k}^{t}\right)^{T} Q_{k} X_{k}^{t}+\left(\mathbb{E}_{t} X_{k}^{t}\right)^{T} \bar{Q}_{k} \mathbb{E}_{t} X_{k}^{t}+u_{k}^{T} R_{k} u_{k}\right. \\ & \left.+\left(\mathbb{E}_{t} u_{k}\right)^{T} \bar{R}_{k} \mathbb{E}_{t} u_{k}\right]+\mathbb{E}_{t}\left[\left(X_{N}^{t}\right)^{T} G X_{N}^{t}\right]+\left(\mathbb{E}_{t} X_{N}^{t}\right)^{T} \bar{G} \mathbb{E}_{t} X_{N}^{t},\end{aligned}$

where $Q_{k}, \bar{Q}_{k}, R_{k}, \bar{R}_{k}, k \in \mathbb{T}_{t}, G, \bar{G}$ are deterministic symmetric matrices of appropriate dimensions.

In $(2), \mathbb{E}_{t}$ is the conditional mathematical expectation $\mathbb{E}\left[\cdot \mid \mathcal{F}_{t}\right]$ with respect to $\mathcal{F}_{t}=\left\{x_{0}, w_{l}, l=0,1, \ldots, t-1\right\}$ and $\mathcal{F}_{0}$ being understood as $\{\emptyset, \Omega\}$. Furthermore, in (1), $x$ is in $L_{\mathcal{F}}^{2}\left(t ; \mathbb{R}^{n}\right)$, which is a set of the random variables such that any $\xi \in L_{\mathcal{F}}^{2}\left(t ; \mathbb{R}^{n}\right)$ is $\mathcal{F}_{t}$-measurable and $\mathbb{E}|\xi|^{2}<\infty$. The noise $\left\{w_{k}, k \in \mathbb{T}\right\}$ is assumed to be a martingale difference sequence defined on a probability space $(\Omega, \mathcal{F}, P)$ in the sense that

$$
\mathbb{E}_{k+1}\left[w_{k+1}\right]=0, \quad \mathbb{E}_{k+1}\left[\left(w_{k+1}\right)^{2}\right]=1, k \geq 0
$$

Let $L_{\mathcal{F}}^{2}\left(\mathbb{T}_{t} ; \mathbb{R}^{m}\right)$ be a space of $\mathbb{R}^{m}$-valued processes such that for any $\nu=$ $\left\{\nu_{k}, k \in \mathbb{T}_{t}\right\} \in L_{\mathcal{F}}^{2}\left(\mathbb{T}_{t} ; \mathcal{H}\right), \nu_{k}$ is $\mathcal{F}_{k}$-measurable and $\sum_{k=t}^{N-1} \mathbb{E}\left|\nu_{k}\right|^{2}<\infty$.

Problem (LQ). Considering (1)(2) and the initial pair $(t, x)$, find $a u^{*} \in$ $L_{\mathcal{F}}^{2}\left(\mathbb{T}_{t} ; \mathbb{R}^{m}\right)$, such that

$$
J\left(t, x ; u^{*}\right)=\inf _{u \in L_{\mathcal{F}}^{2}\left(\mathbb{T}_{t} ; \mathbb{R}^{m}\right)} J(t, x ; u) .
$$

For the feature of time-inconsistency of Problem (LQ), the notion "optimality" should be defined in an appropriate way. Instead of solving Problem (LQ) for the static pre-committed optimal control, we adopt the concept of dynamic equilibrium control, which is optimal in an "infinitesimal" manner and is consistent with the dynamical nature of Problem (LQ).

Definition 1. Given $t \in \mathbb{T}, x \in L_{\mathcal{F}}^{2}\left(t ; \mathbb{R}^{n}\right)$, a state-control pair $\left(X^{t, x, *}, u^{t, x, *}\right)$ $\in L_{\mathcal{F}}^{2}\left(\widetilde{\mathbb{T}}_{t} ; \mathbb{R}^{n}\right) \times L_{\mathcal{F}}^{2}\left(\mathbb{T}_{t} ; \mathbb{R}^{m}\right)$ is called an open-loop equilibrium pair of Problem (LQ) for the initial pair $(t, x)$ if $X_{t}^{t, x, *}=x$, and

$$
J\left(k, X_{k}^{t, x, *} ;\left.u^{t, x, *}\right|_{\mathbb{T}_{k}}\right) \leq J\left(k, X_{k}^{t, x, *} ;\left(u_{k},\left.u^{t, x, *}\right|_{\mathbb{T}_{k+1}}\right)\right)
$$


holds for any $k \in \mathbb{T}_{t}$ and any $u_{k} \in L_{\mathcal{F}}^{2}\left(k ; \mathbb{R}^{m}\right)$. Here, $\left.u^{t, x, *}\right|_{\mathbb{T}_{k}}$ and $\left.u^{t, x, *}\right|_{\mathbb{T}_{k+1}}$ are the restrictions of $u^{t, x, *}$ on $\mathbb{T}_{k}$ and $\mathbb{T}_{k+1}$, respectively, and

$$
\left\{\begin{array}{l}
X_{k+1}^{t, x, *}=\left(A_{k} X_{k}^{t, x, *}+B_{k} u_{k}^{t, x, *}\right)+\left(C_{k} X_{k}^{t, x, *}+D_{k} u_{k}^{t, x, *}\right) w_{k}, \\
X_{t}^{t, x, *}=x, \quad k \in \mathbb{T}_{t}
\end{array}\right.
$$

Furthermore, such a $u^{t, x, *}$ is called an open-loop equilibrium control for the initial pair $(t, x)$.

Note that $\left.u^{t, x, *}\right|_{\mathbb{T}_{k+1}}$ appears in both sides of (5). For any given $k \in \mathbb{T}_{t}$, we denote $J\left(k, X_{k}^{t, x, *} ;\left(u_{k},\left.u^{t, x, *}\right|_{\mathbb{T}_{k+1}}\right)\right)$ by $\bar{J}\left(k, X_{k}^{t, x, *} ; u_{k}\right)$, i.e.,

$$
\bar{J}\left(k, X_{k}^{t, x, *} ; u_{k}\right)=J\left(k, X_{k}^{t, x, *} ;\left(u_{k},\left.u^{t, x, *}\right|_{\mathbb{T}_{k+1}}\right)\right) .
$$

Hence, (5) implies

$$
\bar{J}\left(k, X_{k}^{t, x, *} ; u_{k}^{t, x, *}\right) \leq \bar{J}\left(k, X_{k}^{t, x, *} ; u_{k}\right), \quad \forall u_{k} \in L_{\mathcal{F}}^{2}\left(k ; \mathbb{R}^{m}\right), \quad \forall k \in \mathbb{T}_{t} .
$$

To proceed, review an inner product of $L_{\mathcal{F}}^{2}\left(\mathbb{T}_{t} ; \mathbb{R}^{p}\right)(p=n, m, t \in \mathbb{T})$

$$
\langle y, z\rangle_{\mathbb{T}_{t}}=\sum_{k=t}^{N-1} \mathbb{E}\left(y_{k}^{T} z_{k}\right), \quad y, z \in L_{\mathcal{F}}^{2}\left(\mathbb{T}_{t} ; \mathbb{R}^{p}\right)
$$

and $\langle y, z\rangle_{t}=\mathbb{E}\left(y^{T} z\right)$, for $y, z \in L_{\mathcal{F}}^{2}\left(t ; \mathbb{R}^{p}\right)$, denotes an inner product of $L_{\mathcal{F}}^{2}\left(t ; \mathbb{R}^{p}\right)(p=n, m, t \in \mathbb{T})$. Then $\bar{J}\left(k, X_{k}^{t, x, *} ; u_{k}\right)$ can be represented as

$$
\begin{aligned}
\bar{J}\left(k, X_{k}^{t, x, *} ; u_{k}\right)= & \left\langle Q X^{k}, X^{k}\right\rangle_{\mathbb{T}_{k}}+\left\langle\bar{Q} \mathbb{E}_{k} X^{k}, \mathbb{E}_{k} X^{k}\right\rangle_{\mathbb{T}_{k}} \\
& +\left\langle R_{k} u_{k}, u_{k}\right\rangle_{k}+\left\langle\bar{R}_{k} \mathbb{E}_{k} u_{k}, \mathbb{E}_{k} u_{k}\right\rangle_{k} \\
& +\left\langle R u^{t, x, *}, u^{t, x, *}\right\rangle_{\mathbb{T}_{k+1}}+\left\langle\bar{R} \mathbb{E}_{k} u^{t, x, *}, \mathbb{E}_{k} u^{t, x, *}\right\rangle_{\mathbb{T}_{k+1}} \\
& +\left\langle G X_{N}^{k}, X_{N}^{k}\right\rangle_{N}+\left\langle\bar{G} \mathbb{E}_{k} X_{N}^{k}, \mathbb{E}_{k} X_{N}^{k}\right\rangle_{N}
\end{aligned}
$$

In (9), $X^{k}$ is the solution of the following equation

$$
\left\{\begin{array}{l}
X_{\ell+1}^{k}=\left(A_{\ell} X_{\ell}^{k}+B_{\ell} u_{\ell}^{t, x, *}\right)+\left(C_{\ell} X_{\ell}^{k}+D_{\ell} u_{\ell}^{t, x, *}\right) w_{\ell}, \quad \ell \in \mathbb{T}_{k+1} \\
X_{\ell+1}^{k}=\left(A_{k} X_{k}^{k}+B_{k} u_{k}\right)+\left(C_{k} X_{k}^{k}+D_{k} u_{k}\right) w_{k} \\
X_{k}^{k}=X_{k}^{t, x, *}
\end{array}\right.
$$

$\left\langle Q X^{k}, X^{k}\right\rangle_{\mathbb{T}_{k}}$ and $\left\langle\bar{R} \mathbb{E}_{k} u^{t, x, *}, \mathbb{E}_{k} u^{t, x, *}\right\rangle_{\mathbb{T}_{k+1}}$ represent, respectively,

$$
\sum_{\ell=k}^{N-1} \mathbb{E}_{k}\left[\left(X_{\ell}^{k}\right)^{T} Q_{\ell} X_{\ell}^{k}\right] \text { and } \sum_{\ell=k+1}^{N-1}\left[\left(\mathbb{E}_{k} u_{\ell}^{t, x, *}\right)^{T} \bar{R}_{\ell} \mathbb{E}_{k} u_{\ell}^{t, x, *}\right]
$$

and similar meaning holds for other terms. 
To characterize the open-loop equilibrium control, we now calculate the first order and second order directional derivatives of $\bar{J}\left(k, X_{k}^{t, x, *} ; u_{k}\right)$ at $u_{k}^{t, x, *}$ (when they exist). With the initial pair $\left(k, X_{k}^{t, x, *}\right)$ and the control $\left(u_{k}^{t, x, *}+\lambda \bar{u}_{k},\left.u^{t, x, *}\right|_{\mathbb{T}_{k+1}}\right)$, the solution to (1) is denoted by $X^{t, \lambda}$, where $\bar{u}_{k} \in L_{\mathcal{F}}^{2}\left(k ; \mathbb{R}^{m}\right)$. Then, we have

$$
\left\{\begin{array}{l}
\frac{X_{\ell+1}^{k, \lambda}-X_{\ell+1}^{t, x, *}}{\lambda}=A_{\ell} \frac{X_{\ell}^{k, \lambda}-X_{\ell}^{t, x, *}}{\lambda}+C_{\ell} \frac{X_{\ell}^{k, \lambda}-X_{\ell}^{t, x, *}}{\lambda} w_{\ell}, \quad \ell \in \mathbb{T}_{k+1}, \\
\frac{X_{k+1}^{k, \lambda}-X_{k+1}^{t, x, *}}{\lambda}=\left(A_{k} \frac{X_{k}^{k, \lambda}-X_{k}^{t, x, *}}{\lambda}+B_{k} \bar{u}_{k}\right)+\left(C_{k} \frac{X_{k}^{k, \lambda}-X_{k}^{t, x, *}}{\lambda}+D_{k} \bar{u}_{k}\right) w_{k}, \\
\frac{X_{k}^{k, \lambda}-X_{k}^{t, x, *}}{\lambda}=0 .
\end{array}\right.
$$

Letting $Y_{\ell}^{k}=\frac{X_{\ell}^{k, \lambda}-X_{\ell}^{t, x, *}}{\lambda}$, one gets

$$
\left\{\begin{array}{l}
Y_{\ell+1}^{k}=A_{\ell} Y_{\ell}^{k}+C_{\ell} Y_{\ell}^{k} w_{\ell}, \quad \ell \in \mathbb{T}_{k+1}, \\
Y_{k+1}^{k}=B_{k} \bar{u}_{k}+D_{k} \bar{u}_{k} w_{k}, \\
Y_{k}^{k}=0,
\end{array}\right.
$$

and $X_{\ell}^{k, \lambda}=X_{\ell}^{t, x, *}+\lambda Y_{\ell}^{k}, \ell \in \widetilde{\mathbb{T}}_{k}$. To get the first order directional derivative, we need some calculations. Note that

$$
\begin{aligned}
& \lim _{\lambda \downarrow 0} \frac{\left\langle R_{k}\left(u_{k}^{t, x, *}+\lambda \bar{u}_{k}\right), u_{k}^{t, x, *}+\lambda \bar{u}_{k}\right\rangle_{k}-\left\langle R_{k} u_{k}^{t, x, *}, u_{k}^{t, x, *}\right\rangle_{k}}{\lambda} \\
= & 2\left\langle R_{k} u_{k}^{t, x, *}, \bar{u}_{k}\right\rangle_{t}+\lim _{\lambda \downarrow 0} \lambda\left\langle R_{k} \bar{u}_{k}, \bar{u}_{k}\right\rangle_{k}=2\left\langle R_{k} u_{k}^{t, x, *}, \bar{u}_{k}\right\rangle_{k},
\end{aligned}
$$

and

$$
\begin{aligned}
& \lim _{\lambda \downarrow 0} \frac{\left\langle\bar{R}_{k}\left(\mathbb{E}_{k} u_{k}^{t, x, *}+\lambda \mathbb{E}_{k} \bar{u}_{k}\right), \mathbb{E}_{k} u_{k}^{t, x, *}+\lambda \mathbb{E}_{k} \bar{u}_{k}\right\rangle_{k}-\left\langle\bar{R}_{k} \mathbb{E}_{k} u_{k}^{t, x, *}, \mathbb{E}_{k} u_{k}^{t, x, *}\right\rangle_{k}}{\lambda} \\
= & 2\left\langle\bar{R}_{k} \mathbb{E}_{k} u_{k}^{t, x, *}, \mathbb{E}_{k} \bar{u}_{k}\right\rangle_{k}=2\left\langle\bar{R}_{k} u_{k}^{t, x, *}, \bar{u}_{k}\right\rangle_{k}, \\
& \lim _{\lambda \downarrow 0} \frac{\left\langle Q X^{k, \lambda}, X^{k, \lambda}\right\rangle_{\mathbb{T}_{k}}-\left\langle Q X^{t, x, *}, X^{t, x, *}\right\rangle_{\mathbb{T}_{k}}}{\lambda} \\
= & 2\left\langle Q X^{t, x, *}, Y^{k}\right\rangle_{\mathbb{T}_{k}}, \\
& \lim _{\lambda \downarrow 0} \frac{\left\langle\bar{Q} \mathbb{E}_{k} X^{k, \lambda}, \mathbb{E}_{k} X^{k, \lambda}\right\rangle_{\mathbb{T}_{k}}-\left\langle\bar{Q} \mathbb{E}_{k} X^{t, x, *}, \mathbb{E}_{k} X^{t, x, *}\right\rangle_{\mathbb{T}_{k}}}{\lambda} \\
= & 2\left\langle\bar{Q} \mathbb{E}_{k} X^{t, x, *}, \mathbb{E}_{k} Y^{k}\right\rangle_{\mathbb{T}_{k}}, \\
& \lim _{\lambda \downarrow 0} \frac{\left\langle G X_{N}^{k, \lambda}, X_{N}^{k, \lambda}\right\rangle_{N}-\left\langle G X_{N}^{t, x, *}, X_{N}^{t, x, *}\right\rangle_{N}}{\lambda}
\end{aligned}
$$




$$
\begin{aligned}
= & 2\left\langle G X_{N}^{t, x, *}, Y_{N}^{k}\right\rangle_{N}, \\
& \lim _{\lambda \downarrow 0} \frac{\left\langle\bar{G} \mathbb{E}_{k} X_{N}^{k, \lambda}, \mathbb{E}_{k} X_{N}^{k, \lambda}\right\rangle_{N}-\left\langle\bar{G} \mathbb{E}_{k} X_{N}^{t, x, *}, \mathbb{E}_{k} X_{N}^{t, x, *}\right\rangle_{N}}{\lambda} \\
= & 2\left\langle\bar{G} \mathbb{E}_{k} X_{N}^{t, x, *}, \mathbb{E}_{k} Y_{N}^{k}\right\rangle_{N} .
\end{aligned}
$$

Then, we have the first order directional derivative of $\bar{J}\left(k, X_{k}^{x, *}, u_{k}\right)$ at $u_{k}^{x, *}$ with the direction $\bar{u}_{k}$

$$
\begin{aligned}
& d \bar{J}\left(k, X_{k}^{t, x, *} ; u_{k}^{t, x, *} ; \bar{u}_{k}\right) \\
= & \lim _{\lambda \downarrow 0} \frac{\bar{J}\left(k, X_{k}^{t, x, *} ; u_{k}^{t, x, *}+\lambda \bar{u}_{k}\right)-\bar{J}\left(k, X_{k}^{t, x, *} ; u_{k}^{t, x, *}\right)}{\lambda} \\
= & 2\left\langle Q X^{t, x, *}, Y^{k}\right\rangle_{\mathbb{T}_{k}}+2\left\langle\bar{Q} \mathbb{E}_{k} X^{t, x, *}, \mathbb{E}_{k} Y^{k}\right\rangle_{\mathbb{T}_{k}} \\
& +2\left\langle\left(R_{k}+\bar{R}_{k}\right) u_{k}^{t, x, *}, \bar{u}_{k}\right\rangle_{k} \\
& +2\left\langle G X_{N}^{t, x, *}, Y_{N}^{k}\right\rangle_{N}+2\left\langle\bar{G} \mathbb{E}_{k} X_{N}^{t, x, *}, \mathbb{E}_{k} Y_{N}^{k}\right\rangle_{N} .
\end{aligned}
$$

Another round of lengthy calculations yield the second order directional derivative with the directions $\bar{u}_{k}$ and $\hat{u}_{k}$

$$
\begin{aligned}
& d^{2} \bar{J}\left(k, X_{k}^{t, x, *} ; u_{k}^{t, x, *} ; \bar{u}_{k} ; \hat{u}_{k}\right) \\
= & \lim _{\beta \downarrow 0} \frac{d \bar{J}\left(k, X_{k}^{t, x, *} ; u_{k}^{t, x, *}+\beta \hat{u}_{k} ; \bar{u}_{k}\right)-d \bar{J}\left(k, X_{k}^{t, x, *} ; u_{k}^{t, x, *} ; \bar{u}_{k}\right)}{\beta} \\
= & 2\left\langle Q \hat{Y}^{k}, Y^{k}\right\rangle_{\mathbb{T}_{k}}+2\left\langle\bar{Q} \mathbb{E}_{k} \hat{Y}^{k}, \mathbb{E}_{k} Y^{k}\right\rangle_{\mathbb{T}_{k}}+2\left\langle\left(R_{k}+\bar{R}_{k}\right) \hat{u}_{k}, \bar{u}_{k}\right\rangle_{k} \\
& +2\left\langle G \hat{Y}_{N}^{k}, Y_{N}^{k}\right\rangle_{N}+2\left\langle\bar{G} \mathbb{E}_{k} \hat{Y}_{N}^{k}, \mathbb{E}_{k} Y_{N}^{k}\right\rangle_{N},
\end{aligned}
$$

where

$$
\left\{\begin{array}{l}
\hat{Y}_{\ell+1}^{k}=A_{\ell} \hat{Y}_{\ell}^{k}+C_{\ell} \hat{Y}_{\ell}^{k} w_{\ell}, \quad \ell \in \mathbb{T}_{k+1} \\
\hat{Y}_{k+1}^{k}=B_{k} \hat{u}_{k}+D_{k} \hat{u}_{k} w_{k} \\
\hat{Y}_{k}^{k}=0
\end{array}\right.
$$

If $\hat{u}_{k}=\bar{u}_{k}$, then

$$
\begin{aligned}
d^{2} \bar{J}\left(k, X_{k}^{t, x, *} ; u_{k}^{t, x, *} ; \bar{u}_{k} ; \bar{u}_{k}\right)= & 2\left\langle Q Y^{k}, Y^{k}\right\rangle_{\mathbb{T}_{k}}+2\left\langle\bar{Q} \mathbb{E}_{k} Y^{k}, \mathbb{E}_{k} Y^{k}\right\rangle_{\mathbb{T}_{k}} \\
& +2\left\langle\left(R_{k}+\bar{R}_{k}\right) \bar{u}_{k}, \bar{u}_{k}\right\rangle_{k} \\
& +2\left\langle G Y_{N}^{k}, Y_{N}^{k}\right\rangle_{N}+2\left\langle\bar{G} \mathbb{E}_{k} Y_{N}^{k}, \mathbb{E}_{k} Y_{N}^{k}\right\rangle_{N}
\end{aligned}
$$


Note that the right side of (13) is independent of $u_{k}^{t, x, *}$. Then, for any $u_{k} \in$ $L_{\mathcal{F}}^{2}\left(k ; \mathbb{R}^{m}\right)$ we have

$$
\begin{aligned}
d^{2} \bar{J}\left(k, X_{k}^{t, x, *} ; u_{k} ; \bar{u}_{k} ; \bar{u}_{k}\right)= & 2\left\langle Q Y^{k}, Y^{k}\right\rangle_{\mathbb{T}_{k}}+2\left\langle\bar{Q} \mathbb{E}_{k} Y^{k}, \mathbb{E}_{k} Y^{k}\right\rangle_{\mathbb{T}_{k}} \\
& +2\left\langle\left(R_{k}+\bar{R}_{k}\right) \bar{u}_{k}, \bar{u}_{k}\right\rangle_{k} \\
& +2\left\langle G Y_{N}^{k}, Y_{N}^{k}\right\rangle_{N}+2\left\langle\bar{G} \mathbb{E}_{k} Y_{N}^{k}, \mathbb{E}_{k} Y_{N}^{k}\right\rangle_{N}
\end{aligned}
$$

Furthermore, we could show that $\bar{J}\left(k, X_{k}^{t, x, *} ; u_{k}\right)$ is infinitely differentiable in the sense that the directional derivatives of all orders exist. By classical results on convex analysis [4], we know that the convexity of the map $u_{k} \mapsto \bar{J}\left(k, X_{k}^{t, x, *} ; u_{k}\right)$ is fully characterized via $d^{2} \bar{J}\left(k, X_{k}^{t, x, *} ; u_{k}^{t, x, *} ; \bar{u}_{k} ; \bar{u}_{k}\right) \geq$ 0 . Therefore, we have the following result.

Lemma 2. The following statements are equivalent.

(i) The map $u_{k} \mapsto \bar{J}\left(k, X_{k}^{t, x, *} ; u_{k}\right)$ is convex.

(ii) It holds that

$$
\begin{aligned}
\inf _{\bar{u}_{k} \in L_{\mathcal{F}}^{2}\left(k ; \mathbb{R}^{m}\right)}\left[\left\langle Q Y^{k}, Y^{k}\right\rangle_{\mathbb{T}_{k}}+\left\langle\bar{Q} \mathbb{E}_{k} Y^{k}, \mathbb{E}_{k} Y^{k}\right\rangle_{\mathbb{T}_{k}}+\left\langle\left(R_{k}+R_{k}\right) \bar{u}_{k}, \bar{u}_{k}\right\rangle_{k}\right. \\
\left.+\left\langle G Y_{N}^{k}, Y_{N}^{k}\right\rangle_{N}+\left\langle\bar{G} \mathbb{E}_{k} Y_{N}^{k}, \mathbb{E}_{k} Y_{N}^{k}\right\rangle_{N}\right] \geq 0
\end{aligned}
$$

Theorem 3. Given $t \in \mathbb{T}, x \in L_{\mathcal{F}}^{2}\left(t ; \mathbb{R}^{n}\right)$, the following statements are equivalent.

(i) There exists an open-loop equilibrium pair of Problem (LQ) for the initial pair $(t, x)$.

(ii) There exists a $u^{t, x, *} \in L_{\mathcal{F}}^{2}\left(\mathbb{T}_{t} ; \mathbb{R}^{m}\right)$ such that for any $k \in \mathbb{T}_{t}$, the following FBS $\triangle E$ admits a solution $\left(X^{k, t, x}, Z^{k, t, x}\right)$

$$
\left\{\begin{array}{l}
X_{\ell+1}^{k, t, x}=\left(A_{\ell} X_{\ell}^{k, t, x}+B_{\ell} u_{\ell}^{t, x, *}\right)+\left(C_{\ell} X_{\ell}^{k, t, x}+D_{\ell} u_{\ell}^{t, x, *}\right) w_{\ell} \\
Z_{\ell}^{k, t, x}=A_{\ell}^{T} \mathbb{E}_{\ell} Z_{\ell+1}^{k, t, x}+C_{\ell}^{T} \mathbb{E}_{\ell}\left(Z_{\ell+1}^{k, t, x} w_{\ell}\right)+Q_{\ell} X_{\ell}^{k, t, x}+\bar{Q}_{\ell} \mathbb{E}_{k} X_{\ell}^{k, t, x} \\
X_{k}^{k, t, x}=X_{k}^{t, x, *}, \quad Z_{N}^{k, t, x}=G X_{N}^{k, t, x}+\bar{G} \mathbb{E}_{k} X_{N}^{k, t, x}, \quad \ell \in \mathbb{T}_{k}
\end{array}\right.
$$

with the stationary condition

$$
0=\left(R_{k}+\bar{R}_{k}\right) u_{k}^{t, x, *}+B_{k}^{T} \mathbb{E}_{k} Z_{k+1}^{k, t, x}+D_{k}^{T} \mathbb{E}_{k}\left(Z_{k+1}^{k, t, x} w_{k}\right)
$$

and the convexity condition 


$$
\begin{gathered}
\inf _{\bar{u}_{k} \in L_{\mathcal{F}}^{2}\left(k ; \mathbb{R}^{m}\right)}\left[\left\langle Q Y^{k}, Y^{k}\right\rangle_{\mathbb{T}_{k}}+\left\langle\bar{Q} \mathbb{E}_{k} Y^{k}, \mathbb{E}_{k} Y^{t}\right\rangle_{\mathbb{T}_{k}}+\left\langle\left(R_{k}+R_{k}\right) \bar{u}_{k}, \bar{u}_{k}\right\rangle_{k}\right. \\
\left.+\left\langle G Y_{N}^{k}, Y_{N}^{k}\right\rangle_{N}+\left\langle\bar{G} \mathbb{E}_{k} Y_{N}^{k}, \mathbb{E}_{k} Y_{N}^{k}\right\rangle_{N}\right] \geq 0
\end{gathered}
$$

In the above, $X^{t, x, *}$ is given by

$$
\left\{\begin{array}{l}
X_{k+1}^{t, x, *}=\left(A_{k} X_{k}^{t, x, *}+B_{k} u_{k}^{t, x, *}\right)+\left(C_{k} X_{k}^{t, x, *}+D_{k} u_{k}^{t, x, *}\right) w_{k}, \\
X_{t}^{t, x, *}=x, \quad k \in \mathbb{T}_{t}
\end{array}\right.
$$

In this case, $u^{t, x, *}$ given in (ii) is an open-loop equilibrium control.

Proof. (i) $\Rightarrow$ (ii). Let $\left(X^{t, x, *}, u^{t, x, *}\right)$ be an open-loop equilibrium pair of Problem (LQ) for the initial pair $(t, x)$. Noting that (14) is independent of $u_{k}$, the minimizing point $u_{k}^{t, x, *}$ of $\bar{J}\left(k, X_{k}^{t, x, *} ; u_{k}\right)$ is characterized by the first and second order derivatives: $d \bar{J}\left(k, X_{k}^{t, x, *} ; u_{k}^{t, x, *} ; \bar{u}_{k}\right)=0$ and $d^{2} \bar{J}\left(k, X_{k}^{t, x, *} ; u_{k}^{t, x, *}\right.$; $\left.\bar{u}_{k} ; \bar{u}_{k}\right) \geq 0$ for any $\bar{u}_{k}$ in $L_{\mathcal{F}}^{2}\left(k ; \mathbb{R}^{m}\right)$. Due to this and Lemma 2 , (15) follows. The forward $\mathrm{S} \Delta \mathrm{E}$ about $X^{k, t, x}$ in (16) is solvable as $Z^{k, t, x}$ does not appear in this $\mathrm{S} \Delta \mathrm{E}$. After obtaining $X^{k, t, x}$ and substituting it into the backward $\mathrm{S} \Delta \mathrm{E}$ in (16), we then have $Z^{k, t, x}$. This implies that the FBS $\Delta \mathrm{E}(16)$ admits an adapted solution $\left(X^{k, t, x}, Z^{k, t, x}\right)$.

Taking expectations in both sides of the backward $\mathrm{S} \Delta \mathrm{E}$, we have

$$
\begin{aligned}
Z_{\ell}^{k, t, x}-\mathbb{E}_{k} Z_{\ell}^{k, t, x}= & Q_{\ell}\left(X_{\ell}^{k, t, x}-\mathbb{E}_{k} X_{\ell}^{k, t, x}\right)+A_{\ell}^{T}\left(\mathbb{E}_{\ell} Z_{\ell+1}^{k, t, x}-\mathbb{E}_{k} Z_{\ell+1}^{k, t, x}\right) \\
& +C_{\ell}^{T}\left(\mathbb{E}_{\ell}\left(Z_{\ell+1}^{k, t, x} w_{\ell}\right)-\mathbb{E}_{k}\left(Z_{\ell+1}^{k, t, x} w_{\ell}\right)\right)
\end{aligned}
$$

and

$$
\mathbb{E}_{k} Z_{\ell}^{k, t, x}=\left(Q_{\ell}+\bar{Q}_{\ell}\right) \mathbb{E}_{k} X_{\ell}^{k, t, x}+A_{\ell}^{T} \mathbb{E}_{k} Z_{\ell+1}^{k, t, x}+C_{\ell}^{T} \mathbb{E}_{k}\left(Z_{\ell+1}^{k, t, x} w_{\ell}\right)
$$

Similarly, we have

$$
\left\{\begin{array}{l}
\mathbb{E}_{k} Y_{\ell+1}^{k}=A_{\ell} \mathbb{E}_{k} Y_{\ell}^{k}, \quad \ell \in \mathbb{T}_{k+1}, \\
\mathbb{E}_{k} Y_{k+1}^{k}=B_{k} \bar{u}_{k}, \\
\mathbb{E}_{k} Y_{k}^{k}=0
\end{array}\right.
$$

and

$$
\left\{\begin{array}{l}
Y_{\ell+1}^{k}-\mathbb{E}_{k} Y_{\ell+1}^{k}=A_{\ell}\left(Y_{\ell}^{k}-\mathbb{E}_{k} Y_{\ell}^{k}\right)+\left[C_{\ell}\left(Y_{\ell}^{k}-\mathbb{E}_{k} Y_{\ell}^{k}\right)+C_{\ell} \mathbb{E}_{k} Y_{\ell}^{k}\right] w_{\ell} \\
Y_{k+1}^{k}-\mathbb{E}_{k} Y_{k+1}^{k}=D_{k} \bar{u}_{k} w_{k}, \\
Y_{k}^{k}-\mathbb{E}_{k} Y_{k}^{k}=0, \quad \ell \in \mathbb{T}_{k+1} .
\end{array}\right.
$$


Furthermore, by (12) one can get

$$
\begin{aligned}
& \frac{1}{2} d \bar{J}\left(k, X_{k}^{t, x, *} ; u_{k}^{t, x, *} ; \bar{u}_{k}\right) \\
= & \sum_{\ell=k}^{N-1} \mathbb{E}_{k}\left[\left(Q_{\ell}\left(X_{\ell}^{k, t, x}-\mathbb{E}_{k} X_{\ell}^{k, t, x}\right)\right)^{T}\left(Y_{\ell}^{k}-\mathbb{E}_{k} Y_{\ell}^{k}\right)\right. \\
& \left.+\left(\left(Q_{\ell}+\bar{Q}_{\ell}\right) \mathbb{E}_{k} X_{\ell}^{k, t, x}\right)^{T} \mathbb{E}_{k} Y_{\ell}^{k}\right] \\
& +\mathbb{E}_{k}\left[\left(G\left(X_{N}^{k, t, x}-\mathbb{E}_{k} X_{N}^{k, t, x}\right)\right)^{T}\left(Y_{N}^{k}-\mathbb{E}_{k} Y_{N}^{k}\right)\right. \\
& \left.+\left((G+\bar{G}) \mathbb{E}_{k} X_{N}^{k, t, x}\right)^{T} \mathbb{E}_{k} Y_{N}^{k}\right]+\left[\left(R_{k}+\bar{R}_{k}\right) u_{k}^{t, x, *}\right]^{T} \bar{u}_{k} \\
& N-1 \\
& \sum_{\ell=k} \mathbb{E}_{k}\left[\left(Q_{\ell}\left(X_{\ell}^{k, t, x}-\mathbb{E}_{k} X_{\ell}^{k, t, x}\right)+A_{\ell}^{T}\left(\mathbb{E}_{\ell} Z_{\ell+1}^{k, t, x}-\mathbb{E}_{k} Z_{\ell+1}^{k, t, x}\right)\right.\right. \\
& +C_{\ell}^{T}\left(\mathbb{E}_{\ell}\left(Z_{\ell+1}^{k, t, x} w_{\ell}\right)-\mathbb{E}_{k}\left(Z_{\ell+1}^{k, t, x} w_{\ell}\right)\right) \\
& \left.-\left(Z_{\ell}^{k, t, x}-\mathbb{E}_{k} Z_{\ell}^{k, t, x}\right)\right)^{T}\left(Y_{\ell}^{k}-\mathbb{E}_{k} Y_{\ell}^{k}\right) \\
& +\left(\left(Q_{\ell}+\bar{Q}_{\ell}\right) \mathbb{E}_{k} X_{\ell}^{k, t, x}+A_{\ell}^{T} \mathbb{E}_{k} Z_{\ell+1}^{k, t, x}\right. \\
& \left.\left.+C_{\ell}^{T} \mathbb{E}_{k}\left(Z_{\ell+1}^{k, t, x} w_{\ell}\right)-\mathbb{E}_{k} Z_{\ell}^{k, t, x}\right)^{T} \mathbb{E}_{k} Y_{\ell}^{k}\right] \\
& +\left[\left(R_{k}+\bar{R}_{k}\right) u_{k}^{t, x, *}+B_{k}^{T} \mathbb{E}_{k} Z_{k+1}^{k, t, x}+D_{k}^{T} \mathbb{E}_{k}\left(Z_{k+1}^{k, t, x} w_{k}\right)\right]^{T} \bar{u}_{k} .
\end{aligned}
$$

As $d \bar{J}\left(k, X_{k}^{t, x, *} ; u_{k}^{t, x, *} ; \bar{u}_{k}\right)=0$ for all $\bar{u}_{k} \in L_{\mathcal{F}}^{2}\left(k ; \mathbb{R}^{m}\right)$, we have the stationary condition (17).

(ii) $\Rightarrow($ i). Noting (19) and the solvability of (16) and (17), we have

$$
d \bar{J}\left(k, X_{k}^{t, x, *} ; u_{k}^{t, x, *} ; \bar{u}_{k}\right)=0, \quad \forall \bar{u}_{k} \in L_{\mathcal{F}}^{2}\left(k ; \mathbb{R}^{m}\right) .
$$

Combining this and (15), we know that $u_{k}^{t, x, *}$ is the minimizer of $\bar{J}\left(k, X_{k}^{t, x, *}\right.$; $u_{k}$ ) over $L_{\mathcal{F}}^{2}\left(k ; \mathbb{R}^{m}\right)$. This means that (8), or equivalently, (5) holds for $k \in$ $\mathbb{T}_{t}$. Therefore, $u^{t, x, *}$ is an open-loop equilibrium control of Problem (LQ) for the initial pair $(t, x)$. This proves the conclusion.

By [10], for a given matrix $M \in \mathbb{R}^{n \times m}$, there exists a unique matrix in $\mathbb{R}^{m \times n}$ denoted by $M^{\dagger}$ such that

$$
\left\{\begin{array}{l}
M M^{\dagger} M=M, \quad M^{\dagger} M M^{\dagger}=M^{\dagger} \\
\left(M M^{\dagger}\right)^{T}=M M^{\dagger}, \quad\left(M^{\dagger} M\right)^{T}=M^{\dagger} M .
\end{array}\right.
$$


This $M^{\dagger}$ is called the Moore-Penrose inverse of $M$. The following lemma is from [1].

Lemma 4. Let matrices $L, M$ and $N$ be given with appropriate sizes. Then, $L X M=N$ has a solution $X$ if and only if $L L^{\dagger} N M M^{\dagger}=N$. Moreover, the solution of $L X M=N$ can be expressed as $X=L^{\dagger} N M^{\dagger}+Y-L^{\dagger} L Y M M^{\dagger}$, where $Y$ is a matrix with appropriate size.

Theorem 5. The following statements are equivalent.

(i) For any initial pair $(t, x)$, there exists an open-loop equilibrium control of Problem (LQ).

(ii) The coupled constrained LDEs

$$
\left\{\begin{array}{l}
P_{k}=Q_{k}+A_{k}^{T} P_{k+1} A_{k}+C_{k}^{T} P_{k+1} C_{k} \\
\mathcal{P}_{k}=\mathcal{Q}_{k}+A_{k}^{T} \mathcal{P}_{k+1} A_{k}+C_{k}^{T} P_{k+1} C_{k} \\
P_{N}=G, \quad \mathcal{P}_{N}=\mathcal{G} \\
\mathcal{R}_{k}+B_{k}^{T} \mathcal{P}_{k+1} B_{k}+D_{k}^{T} P_{k+1} D_{k} \geq 0 \\
k \in \mathbb{T}
\end{array}\right.
$$

and the coupled constrained GDREs

$$
\left\{\begin{aligned}
& T_{k}= A_{k}^{T} T_{k+1} A_{k}+C_{k}^{T} T_{k+1} C_{k} \\
& \quad-\left[A_{k}^{T}\left(P_{k+1}+T_{k+1}\right) B_{k}+C_{k}^{T}\left(P_{k+1}+T_{k+1}\right) D_{k}\right] \mathcal{W}_{k}^{\dagger} \mathcal{H}_{k} \\
& \mathcal{T}_{k}= A_{k}^{T} \mathcal{T}_{k+1} \mathcal{A}_{k}+\mathcal{C}_{k}^{T} T_{k+1} C_{k} \\
& \quad-\left[A_{k}^{T}\left(\mathcal{P}_{k+1}+\mathcal{T}_{k+1}\right) B_{k}+C_{k}^{T}\left(P_{k+1}+T_{k+1}\right) D_{k}\right] \mathcal{W}_{k}^{\dagger} \mathcal{H}_{k} \\
& T_{N}= 0, \quad \mathcal{T}_{N}=0 \\
& \mathcal{W}_{k} \mathcal{W}_{k}^{\dagger} \mathcal{H}_{k}-\mathcal{H}_{k}=0 \\
& k \in \mathbb{T}
\end{aligned}\right.
$$

are solvable in the sense that $\mathcal{R}_{k}+B_{k}^{T} \mathcal{P}_{k+1} B_{k}+D_{k}^{T} P_{k+1} D_{k} \geq 0$, $\mathcal{W}_{k} \mathcal{W}_{k}^{\dagger} \mathcal{H}_{k}-\mathcal{H}_{k}=0, k \in \mathbb{T}$, hold. In the above, $\mathcal{Q}_{k}=Q_{k}+\bar{Q}_{k}, \mathcal{R}_{k}=R_{k}+$ $\bar{R}_{k}, \mathcal{G}=G+\bar{G}$, and

$$
\left\{\begin{array}{l}
\mathcal{W}_{k}=\mathcal{R}_{k}+B_{k}^{T}\left(\mathcal{P}_{k+1}+\mathcal{T}_{k+1}\right) B_{k}+D_{k}^{T}\left(P_{k+1}+T_{k+1}\right) D_{k} \\
\mathcal{H}_{k}=B_{k}^{T}\left(\mathcal{P}_{k+1}+\mathcal{T}_{k+1}\right) A_{k}+D_{k}^{T}\left(P_{k+1}+T_{k+1}\right) C_{k} \\
k \in \mathbb{T}
\end{array}\right.
$$


Furthermore, an open-loop equilibrium control for the initial pair $(t, x)$ is given by

$$
u_{k}^{t, x, *}=-\mathcal{W}_{k}^{\dagger} \mathcal{H}_{k} X_{k}^{t, x, *}, \quad k \in \mathbb{T}_{t}
$$

where $X^{t, x, *}$ is given in (6).

Proof. (i) $\Rightarrow$ (ii). For any initial pair $(t, x)$, let Problem (LQ) admit an openloop equilibrium control $u^{t, x, *}$. By Theorem 3 , for each $k \in \mathbb{T}_{t}$, the FBS $\Delta \mathrm{E}$ (16) admits a solution with the stationary condition (17) and the convexity condition (18). Furthermore, from the forward $\mathrm{S} \Delta \mathrm{E}$ of (16) and (6), we know that $X_{\ell}^{k, t, x}=X_{\ell}^{t, x, *}, \ell \in \mathbb{T}_{k}$.

Considering Problem (LQ) for the initial pair $(N-1, x)$ with $x \in L^{2}(N-$ $\left.1 ; \mathbb{R}^{n}\right)$, we have

$$
0=\mathcal{W}_{N-1} u_{N-1}^{N-1, x, *}+\mathcal{H}_{N-1} X_{N-1}^{N-1, x, *}
$$

Letting $e_{i}$ be a $\mathbb{R}^{n}$-valued vector with the $i$-th entry being 1 and other entries 0 , we have

$$
0=\mathcal{W}_{N-1}\left(u_{N-1}^{N-1, e_{1}, *}, \ldots, u_{N-1}^{N-1, e_{n}, *}\right)+\mathcal{H}_{N-1}\left(e_{1}, \ldots, e_{n}\right)
$$

Noting that $\left(e_{1}, \ldots, e_{n}\right)$ is the identity matrix and by Lemma 4 , we have $\mathcal{W}_{N-1} \mathcal{W}_{N-1}^{\dagger} \mathcal{H}_{N-1}-\mathcal{H}_{N-1}=0$, and

$$
u_{N-1}^{t, x, *}=-\mathcal{W}_{N-1}^{\dagger} \mathcal{H}_{N-1} X_{N-1}^{t, x, *}
$$

Considering Problem (LQ) for the initial pair $(N-2, x)$ with $x \in L_{\mathcal{F}}^{2}(N-$ $\left.2 ; \mathbb{R}^{n}\right)$, we can similarly prove

$$
\mathcal{W}_{N-2} \mathcal{W}_{N-2}^{\dagger} \mathcal{H}_{N-2}-\mathcal{H}_{N-2}=0
$$

and

$$
u_{N-2}^{t, x, *}=-\mathcal{W}_{N-2}^{\dagger} \mathcal{H}_{N-2} X_{N-2}^{t, x, *} .
$$

Continuing above procedure backwardly, we then have the solvability of (22) and (24). Furthermore, by adding and subtracting 


$$
\begin{aligned}
& \sum_{\ell=k}^{N-1} \mathbb{E}_{k}\left[\left(Y_{\ell+1}^{k}\right)^{T} P_{\ell+1} Y_{\ell+1}^{k}-\left(Y_{\ell}^{k}\right)^{T} P_{\ell} Y_{\ell}^{k}\right. \\
& \left.\quad+\left(\mathbb{E}_{k} Y_{\ell+1}^{k}\right)^{T} \bar{P}_{\ell+1} \mathbb{E}_{k} Y_{\ell+1}^{k}-\left(\mathbb{E}_{k} Y_{\ell}^{k}\right)^{T} \bar{P}_{\ell} \mathbb{E}_{k} Y_{\ell}^{k}\right]
\end{aligned}
$$

we have

$$
\begin{aligned}
& \left\langle Q Y^{k}, Y^{k}\right\rangle_{\mathbb{T}_{k}}+\left\langle\bar{Q} \mathbb{E}_{k} Y^{k}, \mathbb{E}_{k} Y^{t}\right\rangle_{\mathbb{T}_{k}}+\left\langle\left(R_{k}+R_{k}\right) \bar{u}_{k}, \bar{u}_{k}\right\rangle_{k} \\
& +\left\langle G Y_{N}^{k}, Y_{N}^{k}\right\rangle_{N}+\left\langle\bar{G} \mathbb{E}_{k} Y_{N}^{k}, \mathbb{E}_{k} Y_{N}^{k}\right\rangle_{N} \\
= & \sum_{\ell=k}^{N-1} \mathbb{E}_{k}\left[\left(Y_{\ell}^{k}\right)^{T} Q_{\ell} Y_{\ell}^{k}+\left(\mathbb{E}_{k} Y_{\ell}^{k}\right)^{T} \bar{Q}_{\ell} \mathbb{E}_{k} Y_{\ell}^{k}\right]+\bar{u}_{k}^{T} \mathcal{R}_{k} \bar{u}_{k} \\
& +\mathbb{E}_{k}\left[\left(Y_{N}^{k}\right)^{T} G Y_{N}^{k}\right]+\left(\mathbb{E}_{k} Y_{N}^{k}\right)^{T} \bar{G}_{\mathbb{E}_{k} Y_{N}^{k}} \\
= & \sum_{\ell=k}^{N-1} \mathbb{E}_{k}\left[\left(Y_{\ell}^{k}\right)^{T} Q_{\ell} Y_{\ell}^{k}+\left(\mathbb{E}_{k} Y_{\ell}^{k}\right)^{T} \bar{Q}_{\ell} \mathbb{E}_{k} Y_{\ell}^{k}+\left(Y_{\ell+1}^{k}\right)^{T} P_{\ell+1} Y_{\ell+1}^{k}\right. \\
& \left.-\left(Y_{\ell}^{k}\right)^{T} P_{\ell} Y_{\ell}^{k}+\left(\mathbb{E}_{k} Y_{\ell+1}^{k}\right)^{T} \bar{P}_{\ell+1} \mathbb{E}_{k} Y_{\ell+1}^{k}-\left(\mathbb{E}_{k} Y_{\ell}^{k}\right)^{T} \bar{P}_{\ell} \mathbb{E}_{k} Y_{\ell}^{k}\right] \\
& +\bar{u}_{k}^{T} \mathcal{R}_{k} \bar{u}_{k} \\
= & \sum_{\ell=}^{N-1} \mathbb{E}_{k}\left[\left(\mathbb{E}_{k} Y_{\ell}^{k}\right)^{T}\left(\mathcal{Q}_{\ell}+\mathcal{A}_{\ell}^{T} \mathcal{P}_{\ell+1} \mathcal{A}_{\ell}+\mathcal{C}_{\ell}^{T} P_{\ell+1} \mathcal{C}_{\ell}-\mathcal{P}_{\ell}\right) \mathbb{E}_{k} Y_{\ell}^{k}\right. \\
& +k+1 \\
& \left.+\left(Y_{\ell}^{k}-\mathbb{E}_{k} Y_{\ell}^{k}\right)^{T}\left(Q_{\ell}+A_{\ell}^{T} P_{\ell+1} A_{\ell}+C_{\ell}^{T} P_{\ell+1} C_{\ell}-P_{\ell}\right)\left(Y_{\ell}^{k}-\mathbb{E}_{k} Y_{\ell}^{k}\right)\right] \\
& +\bar{u}_{k}^{T}\left(\mathcal{R}_{k}+B_{k}^{T} \mathcal{P}_{k+1} B_{k}+D_{k}^{T} P_{k+1} D_{k}\right) \bar{u}_{k} \\
= & \bar{u}_{k}^{T}\left(\mathcal{R}_{k}+B_{k}^{T} \mathcal{P}_{k+1} B_{k}+D_{k}^{T} P_{k+1} D_{k}\right) \bar{u}_{k} .
\end{aligned}
$$

From (18) and (25), we have $\mathcal{R}_{k}+B_{k}^{T} \mathcal{P}_{k+1} B_{k}+D_{k}^{T} P_{k+1} D_{k} \geq 0$. Thus, (21) is solvable.

(ii) $\Rightarrow($ i). Letting (21) and (22) be solvable, we now prove that for any initial pair $(t, x)$, Problem (LQ) admits an open-loop equilibrium pair.

Introduce a dynamics

$$
\left\{\begin{array}{l}
\widetilde{X}_{k+1}^{t, x, *}=\left(A_{k}-B_{k} \mathcal{W}_{k}^{\dagger} \mathcal{H}_{k}\right) \tilde{X}_{k}^{t, x, *}+\left(A_{k}-B_{k} \mathcal{W}_{k}^{\dagger} \mathcal{H}_{k}\right) \widetilde{X}_{k}^{t, x, *} w_{k} \\
\widetilde{X}_{t}^{t, x, *}=x, \quad k \in \mathbb{T}_{t}
\end{array}\right.
$$

and a control

$$
\widetilde{u}_{k}^{t, x, *}=-\mathcal{W}_{k}^{\dagger} \mathcal{H}_{k} \tilde{X}_{k}^{t, x, *}, \quad k \in \mathbb{T}_{t}
$$


As the backward state does not appear in the forward $\mathrm{S} \Delta \mathrm{E}$, the FBS $\Delta \mathrm{E}$

$$
\left\{\begin{array}{l}
\widetilde{X}_{\ell+1}^{k, t, x}=\left(A_{\ell} \widetilde{X}_{\ell}^{k, t, x}+B_{\ell} \widetilde{u}_{\ell}^{t, x, *}\right)+\left(C_{\ell} \widetilde{X}_{\ell}^{k, t, x}+D_{\ell} \widetilde{u}_{\ell}^{t, x, *}\right) w_{\ell}, \\
\widetilde{Z}_{\ell}^{k, t, x}=A_{\ell}^{T} \mathbb{E}_{\ell} \widetilde{Z}_{\ell+1}^{k, t, x}+C_{\ell}^{T} \mathbb{E}_{\ell}\left(\widetilde{Z}_{\ell+1}^{k, t, x} w_{\ell}\right)+Q_{\ell} \widetilde{X}_{\ell}^{k, t, x}+\bar{Q}_{\ell} \mathbb{E}_{k} \widetilde{X}_{\ell}^{k, t, x}, \\
\widetilde{X}_{k}^{k, t, x}=\widetilde{X}_{k}^{t, x, *}, \quad \widetilde{Z}_{N}^{k, t, x}=G \widetilde{X}_{N}^{k, t, x}+\bar{G}_{\mathbb{E}_{k}} \widetilde{X}_{N}^{k, t, x}, \quad \ell \in \mathbb{T}_{k}
\end{array}\right.
$$

admits an adapted solution. We further have

$$
\widetilde{Z}_{\ell}^{k, t, x}=\left(P_{\ell}+T_{\ell}\right) \widetilde{X}_{\ell}^{k, t, x}+\left(\bar{P}_{\ell}+\bar{T}_{\ell}\right) \mathbb{E}_{k} \widetilde{X}_{\ell}^{k, t, x}, \quad \ell \in \mathbb{T}_{k+1},
$$

where $\bar{P}_{\ell}=\mathcal{P}_{\ell}-P_{\ell}$ and $\bar{T}_{\ell}=\mathcal{T}_{\ell}-T_{\ell}$. In fact, by (26) and (27), we have

$$
\left\{\begin{array}{l}
\widetilde{X}_{k+1}^{t, x, *}=\left(A_{k} \widetilde{X}_{k}^{t, x, *}+B_{k} \widetilde{u}_{k}^{t, x, *}\right)+\left(A_{k} \tilde{X}_{k}^{t, x, *}+B_{k} \widetilde{u}_{k}^{t, x, *}\right) w_{k}, \\
\widetilde{X}_{t}^{t, x, *}=x, \quad k \in \mathbb{T}_{t} .
\end{array}\right.
$$

From $\tilde{X}_{k}^{k, t, x}=\tilde{X}_{k}^{t, x, *}$ it follows that

$$
\widetilde{X}_{\ell}^{k, t, x}=\widetilde{X}_{\ell}^{t, x, *}, \quad \ell \in \mathbb{T}_{k} .
$$

Hence,

$$
\begin{aligned}
& A_{N-1}^{T} \mathbb{E}_{N-1} \widetilde{Z}_{N}^{k, t, x}=A_{N-1}^{T} \mathbb{E}_{N-1}\left[G \widetilde{X}_{N}^{k, t, x}+\bar{G} \mathbb{E}_{k} \widetilde{X}_{N}^{k, t, x}\right] \\
= & A_{N-1}^{T} G A_{N-1} \widetilde{X}_{N-1}^{k, t, x}+A_{N-1}^{T} \bar{G} A_{N-1} \mathbb{E}_{k} \widetilde{X}_{N-1}^{k, t, x} \\
& +A_{N-1}^{T} G B_{N-1} \mathcal{W}_{N-1}^{\dagger} \mathcal{H}_{N-1} \widetilde{X}_{N-1}^{t, x *}+A_{N-1}^{T} \bar{G} B_{N-1} \mathcal{W}_{N-1}^{\dagger} \mathcal{H}_{N-1} \mathbb{E}_{k} \widetilde{X}_{N-1}^{t, x, *},
\end{aligned}
$$

and

$$
\begin{aligned}
C_{N-1}^{T} \mathbb{E}_{N-1}\left(\widetilde{Z}_{N}^{k, t, x} w_{N-1}\right)= & C_{N-1}^{T} G C_{N-1} \tilde{X}_{N-1}^{k, t, x} \\
& +C_{N-1}^{T} G D_{N-1} \mathcal{W}_{N-1}^{\dagger} \mathcal{H}_{N-1} \widetilde{X}_{N-1}^{t, x, *}
\end{aligned}
$$

Therefore, we have

$$
\begin{aligned}
\widetilde{Z}_{N-1}^{k, t, x}= & {\left[Q_{N-1}+A_{N-1}^{T} G A_{N-1}+C_{N-1}^{T} G C_{N-1}\right] \widetilde{X}_{N-1}^{k, t, x} } \\
& +\left[\bar{Q}_{N-1}+A_{N-1}^{T} \bar{G} A_{N-1}\right] \mathbb{E}_{k} \widetilde{X}_{N-1}^{k, t, x} \\
& -\left[A_{N-1}^{T} G B_{N-1}+C_{N-1}^{T} G D_{N-1}\right] \mathcal{W}_{N-1}^{\dagger} \mathcal{H}_{N-1} \widetilde{X}_{N-1}^{t, x, *} \\
& -A_{N-1}^{T} \bar{G} B_{N-1} \mathcal{W}_{N-1}^{\dagger} \mathcal{H}_{N-1} \mathbb{E}_{k} \widetilde{X}_{N-1}^{t, x, *} \\
= & P_{N-1} \widetilde{X}_{N-1}^{k, t, x}+\bar{P}_{N-1} \mathbb{E}_{k} \widetilde{X}_{N-1}^{k, t, x}+T_{N-1} \widetilde{X}_{N-1}^{t, x, *}+\bar{T}_{N-1} \mathbb{E}_{k} \widetilde{X}_{N-1}^{t, x, *}
\end{aligned}
$$




$$
=\left(P_{N-1}+T_{N-1}\right) \widetilde{X}_{N-1}^{k, t, x}+\left(\bar{P}_{N-1}+\bar{T}_{N-1}\right) \mathbb{E}_{k} \widetilde{X}_{N-1}^{k, t, x}
$$

Similarly, we have

$$
\begin{aligned}
\widetilde{Z}_{N-2}^{k, t, x}= & {\left[Q_{N-2}+A_{N-2}^{T} P_{N-1} A_{N-2}+C_{N-2}^{T} P_{N-1} C_{N-2}\right] \widetilde{X}_{N-2}^{k, t, x} } \\
& +\left[\bar{Q}_{N-2}+A_{N-2}^{T} \bar{P}_{N-1} A_{N-2}\right] \mathbb{E}_{k} \widetilde{X}_{N-2}^{k, t, x} \\
& +\left\{A_{N-2}^{T} T_{N-1} A_{N-2}+C_{N-2}^{T} T_{N-1} C_{N-2}\right. \\
& -\left[A_{N-2}^{T}\left(P_{N-1}+T_{N-1}\right) B_{N-2}\right. \\
& \left.\left.+C_{N-2}^{T}\left(P_{N-1}+T_{N-1}\right) D_{N-2}\right] \mathcal{W}_{N-2}^{\dagger} \mathcal{H}_{N-2}\right\} \widetilde{X}_{N-2}^{t, x, *} \\
& +\left[A_{N-2}^{T} \bar{T}_{N-1} A_{N-2}\right. \\
& \left.-A_{N-2}^{T}\left(\bar{P}_{N-1}+\bar{T}_{N-1}\right) B_{N-2} \mathcal{W}_{N-2}^{\dagger} \mathcal{H}_{N-2}\right] \mathbb{E}_{k} \widetilde{X}_{N-2}^{t, x, *} \\
= & P_{N-2} \widetilde{X}_{N-2}^{k, t, x}+\bar{P}_{N-2} \mathbb{E}_{k} \widetilde{X}_{N-2}^{k, t, x}+T_{N-2} \widetilde{X}_{N-2}^{t, x, *}+\bar{T}_{N-2} \mathbb{E}_{k} \widetilde{X}_{N-2}^{t, x, *} \\
= & \left(P_{N-2}+T_{N-2}\right) \widetilde{X}_{N-2}^{k, t, x}+\left(\bar{P}_{N-2}+\bar{T}_{N-2}\right) \mathbb{E}_{k} \widetilde{X}_{N-2}^{k, t, x} .
\end{aligned}
$$

By deduction, we can get (28).

From Lemma 4 and (28), we have

$$
\begin{aligned}
& \mathcal{R}_{k} \widetilde{u}_{k}^{t, x, *}+B_{k}^{T} \mathbb{E}_{k} \widetilde{Z}_{k+1}^{k, t, x}+D_{k}^{T} \mathbb{E}_{k}\left(\widetilde{Z}_{k+1}^{k, t, x} w_{k}\right) \\
& =\mathcal{R}_{k} \widetilde{u}_{k}^{t, x, *}+B_{k}^{T}\left(\mathcal{P}_{k}+\mathcal{T}_{k}\right) \mathbb{E}_{k} \widetilde{X}_{k+1}^{k, t, x}+D_{k}^{T}\left(P_{k}+T_{k}\right) \mathbb{E}_{k}\left(\widetilde{X}_{k+1}^{k, t, x} w_{k}\right) \\
& =0
\end{aligned}
$$

From Theorem 3, Problem (LQ) for the initial pair $(t, x)$ admits an open-loop equilibrium pair, and $\left(\widetilde{X}^{t, x, *}, \widetilde{u}^{t, x, *}\right)$ is an equilibrium pair. This completes the proof.

\section{Closed-loop time-consistent equilibrium strategy}

Let $L^{2}\left(\mathbb{T} ; \mathbb{R}^{m \times n}\right)$ be a set of $\mathbb{R}^{m \times n}$-valued deterministic processes such that for any $\nu=\left\{\nu_{k}, k \in \mathbb{T}\right\} \in L^{2}\left(\mathbb{T} ; \mathbb{R}^{m \times n}\right), \sum_{k=0}^{N-1}\left|\nu_{k}\right|^{2}<\infty$. The following definition is concerned with the time-consistency of the feedback strategy.

Definition 6. $\Phi \in L^{2}\left(\mathbb{T} ; \mathbb{R}^{m \times n}\right)$ is called a closed-loop equilibrium strategy of Problem (LQ) if for any $t \in \mathbb{T}, k \in \mathbb{T}_{t}, x \in L_{\mathcal{F}}^{2}\left(k ; \mathbb{R}^{n}\right)$ and $u_{k} \in L_{\mathcal{F}}^{2}\left(k ; \mathbb{R}^{m}\right)$,

$$
J\left(k, X_{k}^{t, x, *} ;\left.\left(\Phi X^{t, x, *}\right)\right|_{\mathbb{T}_{k}}\right) \leq J\left(k, X_{k}^{t, x, *} ;\left(u_{k},\left.\left(\Phi X^{k, u_{k}, \Phi}\right)\right|_{\mathbb{T}_{k+1}}\right)\right)
$$


holds. Here, $X^{t, x, *}=\left\{X_{k}^{t, x, *}, k \in \widetilde{\mathbb{T}}_{t}\right\}$ and $X^{k, u_{k}, \Phi}=\left\{X_{\ell}^{k, u_{k}, \Phi}, \ell \in \widetilde{\mathbb{T}}_{k}\right\}$ are given by

$$
\begin{aligned}
& \left\{\begin{array}{l}
X_{k+1}^{t, x, *}=\left(A_{k}+B_{k} \Phi_{k}\right) X_{k}^{t, x, *}+\left(C_{k}+D_{k} \Phi_{k}\right) X_{k}^{t, x, *} w_{k}, \quad k \in \mathbb{T}_{t}, \\
X_{t}^{t, x, *}=x
\end{array}\right. \\
& \left\{\begin{array}{l}
X_{\ell+1}^{k, u_{k}, \Phi}=\left(A_{\ell}+B_{\ell} \Phi_{\ell}\right) X_{\ell}^{k, u_{k}, \Phi}+\left(C_{\ell}+D_{\ell} \Phi_{\ell}\right) X_{\ell}^{k, u_{k}, \Phi} w_{\ell}, \\
X_{k+1}^{k, u_{k}, \Phi}=\left(A_{k} X_{k}^{k, u_{k}, \Phi}+B_{k} u_{k}\right)+\left(C_{k} X_{k}^{k, u_{k}, \Phi}+D_{k} u_{k}\right) w_{k}, \\
X_{k}^{k, u_{k}, \Phi}=X_{k}^{t, x, *}, \quad \ell \in \mathbb{T}_{k+1},
\end{array}\right.
\end{aligned}
$$

and $\left.\left(\Phi X^{t, x, *}\right)\right|_{\mathbb{T}_{k}},\left.\left(\Phi X^{k, u_{k}, \Phi}\right)\right|_{\mathbb{T}_{k+1}}$ are defined by

$$
\begin{aligned}
& \left.\left(\Phi X^{t, x, *}\right)\right|_{\mathbb{T}_{k}}=\left(\Phi_{k} X_{k}^{t, x, *}, \ldots, \Phi_{N-1} X_{N-1}^{t, x, *}\right), \\
& \left.\left(\Phi X^{k, u_{k}, \Phi}\right)\right|_{\mathbb{T}_{k+1}}=\left(\Phi_{k+1} X_{k+1}^{k, u_{t}, \Phi}, \ldots, \Phi_{N-1} X_{N-1}^{k, u_{k}, \Phi}\right) .
\end{aligned}
$$

Furthermore, $\Phi$ is required to be independent of all the initial pair.

Though the notation $X^{t, x, *}$ is also used in the previous section, throughout this section $X^{t, x, *}$ will denote the solution of (30). Noting that $\left.\left.\left(\Phi X^{t, x, *}\right)\right|_{\mathbb{T}_{k}}=\left(\Phi_{k} X_{k}^{t, x, *},\left.\left(\Phi X^{t, x, *}\right)\right|_{\mathbb{T}_{k+1}}\right),\left(u_{k},\left.\left(\Phi X^{k, u_{t}, \Phi}\right)\right|_{\mathbb{T}_{k+1}}\right)\right)$ is obtained from $\left.\left(\Phi X^{t, x, *}\right)\right|_{\mathbb{T}_{k}}$ by not only replacing $\Phi_{k} X_{k}^{t, x, *}$ with $u_{k}$ but also replacing $X^{t, x, *}$ in $\left.\left(\Phi X^{t, x, *}\right)\right|_{\mathbb{T}_{k+1}}$ with $X^{k, u_{k}, \Phi}$. This is essentially different from the case of the open-loop formulation. Briefly speaking, here we will focus on the property of the strategy instead of the control.

Theorem 7. The following statements are equivalent.

(i) There exists a closed-loop equilibrium strategy of Problem (LQ).

(ii) There exists a $\Phi \in L^{2}\left(\mathbb{T} ; \mathbb{R}^{n \times m}\right)$ such that for any $t \in \mathbb{T}$, $x \in L_{\mathcal{F}}^{2}\left(\mathbb{T}_{t} ; \mathbb{R}^{n}\right)$ and $k \in \mathbb{T}_{t}$ the following $F B S \Delta E$ admits an adapted solution $\left(X^{k, \Phi}, Z^{k, \Phi}\right)$

$$
\left\{\begin{aligned}
X_{\ell+1}^{k, \Phi}= & \left(A_{\ell}+B_{\ell} \Phi_{\ell}\right) X_{\ell}^{k, \Phi}+\left(C_{\ell}+D_{\ell} \Phi_{\ell}\right) X_{\ell}^{k, \Phi} w_{\ell} \\
Z_{\ell}^{k, \Phi}= & Q_{\ell} X_{\ell}^{k, \Phi}+\bar{Q}_{\ell} \mathbb{E}_{k} X_{\ell}^{k, \Phi}+\Phi_{\ell}^{T} R_{\ell} \Phi_{\ell} X_{\ell}^{k, \Phi}+\Phi_{\ell}^{T} \bar{R}_{\ell} \Phi_{\ell} \mathbb{E}_{k} X_{\ell}^{k, \Phi} \\
& +\left(A_{\ell}+B_{\ell} \Phi_{\ell}\right)^{T} \mathbb{E}_{\ell} Z_{\ell+1}^{k, \Phi}+\left(C_{\ell}+D_{\ell} \Phi_{\ell}\right)^{T} \mathbb{E}_{\ell}\left(Z_{\ell+1}^{k, \Phi} w_{\ell}\right) \\
X_{k}^{k, \Phi}= & X_{k}^{t, x, *}, \quad Z_{N}^{k, \Phi}=G X_{N}^{k, \Phi}+\bar{G}_{k} \mathbb{E}_{N}^{k, \Phi}, \quad \ell \in \mathbb{T}_{k}
\end{aligned}\right.
$$

with the following stationary condition

$$
0=\mathcal{R}_{k} \Phi_{k} X_{k}^{t, x, *}+B_{k}^{T} \mathbb{E}_{k} Z_{k+1}^{k, \Phi}+D_{k}^{T} \mathbb{E}_{k}\left(Z_{k+1}^{k, \Phi} w_{k}\right),
$$


and the convexity condition

$$
\begin{aligned}
& \inf _{\bar{u}_{k} \in L_{\mathcal{F}}^{2}\left(k ; \mathbb{R}^{m}\right)}\left[\left\langle Q Y^{k, \bar{u}_{k}, \Phi}, Y^{k, \bar{u}_{k}, \Phi}\right\rangle_{\mathbb{T}_{k}}+\left\langle\bar{Q} \mathbb{E}_{k} Y^{k, \bar{u}_{k}, \Phi}, \mathbb{E}_{k} Y^{k, \bar{u}_{k}, \Phi}\right\rangle_{\mathbb{T}_{k}}\right. \\
& \quad+\left\langle R_{k} \bar{u}_{k}, \bar{u}_{k}\right\rangle_{k}+\left\langle R \Phi Y^{k, \bar{u}_{k}, \Phi}, \Phi Y^{k, \bar{u}_{k}, \Phi}\right\rangle_{\mathbb{T}_{k+1}} \\
& \quad+\left\langle\bar{R}_{k, k} \mathbb{E}_{k} \bar{u}_{k}, \mathbb{E}_{k} \bar{u}_{k}\right\rangle_{k}+\left\langle\bar{R}\left(\mathbb{E}_{k}\left(\Phi Y^{k, \bar{u}_{k}, \Phi}\right)\right), \mathbb{E}_{k}\left(\Phi Y^{k, \bar{u}_{k}, \Phi}\right)\right\rangle_{\mathbb{T}_{k+1}} \\
& \left.\quad+\left\langle G Y_{N}^{k, \bar{u}_{k}, \Phi}, Y_{N}^{k, \bar{u}_{k}, \Phi}\right\rangle_{N}+\left\langle\bar{G} \mathbb{E}_{k} Y_{N}^{k, \bar{u}_{k}, \Phi}, \mathbb{E}_{k} Y_{N}^{k, \bar{u}_{k}, \Phi}\right\rangle_{N}\right] \geq 0
\end{aligned}
$$

Here, $X^{t, x, *}=\left\{X_{k}^{t, x, *}, k \in \widetilde{\mathbb{T}}_{t}\right\}$ and $Y^{k, \bar{u}_{k}, \Phi}$ are given by

$$
\left\{\begin{array}{l}
X_{k+1}^{t, x, *}=\left(A_{k}+B_{k} \Phi_{k}\right) X_{k}^{t, x, *}+\left(C_{k}+D_{k} \Phi_{k}\right) X_{k}^{t, x, *} w_{k}, \quad k \in \mathbb{T}_{t} \\
X_{t}^{t, x, *}=x
\end{array}\right.
$$

and

$$
\left\{\begin{aligned}
Y_{\ell+1}^{k, \bar{u}_{k}, \Phi} & =\left(A_{\ell}+B_{\ell} \Phi_{\ell}\right) Y_{\ell}^{k, \bar{u}_{k}, \Phi}++\left(C_{\ell}+D_{\ell} \Phi_{\ell}\right) Y_{\ell}^{k, \bar{u}_{k}, \Phi} w_{\ell} \\
Y_{k+1}^{k, \bar{u}_{k}, \Phi} & =B_{k, k} \bar{u}_{k}+D_{k, k} \bar{u}_{k} w_{k} \\
Y_{k}^{k, \bar{u}_{k}, \Phi} & =0, \quad \ell \in \mathbb{T}_{k+1} .
\end{aligned}\right.
$$

In (34), $\left\langle Q Y^{k, \bar{u}_{k}, \Phi}, Y^{k, \bar{u}_{k}, \Phi}\right\rangle_{\mathbb{T}_{k}}$ represents $\sum_{\ell=k}^{N-1} \mathbb{E}_{k}\left[\left(Y_{\ell}^{k, \bar{u}_{k}, \Phi}\right)^{T} Q_{\ell} Y_{\ell}^{k, \bar{u}_{k}, \Phi}\right]$, and similar meaning holds for other terms.

In this case, $\Phi$ in (ii) is a closed-loop equilibrium strategy.

Proof. By some calculations, one can get

$$
\begin{aligned}
& J\left(k, X_{k}^{t, x, *} ;\left(u_{k},\left.\left(\Phi X^{k, u_{k}, \Phi}\right)\right|_{\mathbb{T}_{k+1}}\right)\right) \\
= & \left\langle Q X^{k, u_{k}, \Phi}, X^{k, u_{k}, \Phi}\right\rangle_{\mathbb{T}_{k}}+\left\langle\bar{Q} \mathbb{E}_{k} X^{k, u_{k}, \Phi}, \mathbb{E}_{k} X^{k, u_{k}, \Phi}\right\rangle_{\mathbb{T}_{k}}+\left\langle R_{k} u_{k}, u_{k}\right\rangle_{k} \\
& +\left\langle R \Phi X^{k, u_{k}, \Phi}, \Phi X^{k, u_{k}, \Phi}\right\rangle_{\mathbb{T}_{k+1}}+\left\langle\bar{R}_{k} \mathbb{E}_{k} u_{k}, \mathbb{E}_{k} u_{k}\right\rangle_{k} \\
& +\left\langle\bar{R} \mathbb{E}_{k}\left(\Phi X^{k, u_{k}, \Phi}\right), \mathbb{E}_{k}\left(\Phi X^{k, u_{k}, \Phi}\right)\right\rangle_{\mathbb{T}_{k+1}} \\
& +\left\langle G X_{N}^{k, u_{k}, \Phi}, X_{N}^{k, u_{k}, \Phi}\right\rangle_{N}+\left\langle\bar{G} \mathbb{E}_{k} X_{N}^{k, u_{k}, \Phi}, \mathbb{E}_{k} X_{N}^{k, u_{k}, \Phi}\right\rangle_{N} \\
= & \left\langle Q X^{k, u_{k}, \Phi}, X^{k, u_{k}, \Phi}\right\rangle_{\mathbb{T}_{k}}+\left\langle\bar{Q} \mathbb{E}_{k} X^{k, u_{k}, \Phi}, \mathbb{E}_{k} X^{k, u_{k}, \Phi}\right\rangle_{\mathbb{T}_{k}}+\left\langle R_{k} u_{k}, u_{k}\right\rangle_{k} \\
& +\left\langle R \Phi X^{k, u_{k}, \Phi}, \Phi X^{k, u_{k}, \Phi}\right\rangle_{\mathbb{T}_{k+1}}+\left\langle\bar{R}_{k, k} \mathbb{E}_{k} u_{k}, \mathbb{E}_{k} u_{k}\right\rangle_{k} \\
& +\left\langle\bar{R} \mathbb{E}_{k}\left(\Phi X^{k, u_{k}, \Phi}\right), \mathbb{E}_{k}\left(\Phi X^{k, u_{k}, \Phi}\right)\right\rangle_{\mathbb{T}_{k+1}}
\end{aligned}
$$




$$
+\left\langle G X_{N}^{k, u_{k}, \Phi}, X_{N}^{k, u_{k}, \Phi}\right\rangle_{N}+\left\langle\bar{G} \mathbb{E}_{k} X_{N}^{k, u_{k}, \Phi}, \mathbb{E}_{k} X_{N}^{k, u_{k}, \Phi}\right\rangle_{N}
$$

which is denoted as $\widetilde{J}\left(k, X_{k}^{t, x, *} ; u_{k}\right)$. Similarly, we can get the expression of $J\left(k, X_{k}^{t, x, *} ;\left(u_{k}+\lambda \bar{u}_{k},\left.\left(\Phi X^{k, u_{k}, \bar{u}_{k}, \Phi, \lambda}\right)\right|_{\mathbb{T}_{k+1}}\right)\right) \triangleq \widetilde{J}\left(k, X_{k}^{t, x, *} ; u_{k}+\lambda \bar{u}_{k}\right)$, where

$$
\left\{\begin{aligned}
X_{\ell+1}^{k, u_{k}, \bar{u}_{k}, \Phi, \lambda}= & \left(A_{\ell}+B_{\ell} \Phi_{\ell}\right) X_{\ell}^{k, u_{k}, \bar{u}_{k}, \Phi, \lambda} \\
& +\left(C_{\ell}+D_{\ell} \Phi_{\ell}\right) X_{\ell}^{k, u_{k}, \bar{u}_{k}, \Phi, \lambda} w_{\ell}, \quad \ell \in \mathbb{T}_{k+1}, \\
X_{k+1}^{k, u_{k}, \bar{u}_{k}, \Phi, \lambda}= & A_{k} X_{k}^{k, u_{k}, \bar{u}_{k}, \Phi, \lambda}+\mathcal{B}_{k}\left(u_{k}+\lambda \bar{u}_{k}\right) \\
& +\left[C_{k} X_{k}^{k, u_{k}, \bar{u}_{k}, \Phi, \lambda}+D_{k}\left(u_{k}+\lambda \bar{u}_{k}\right)\right] w_{k}, \\
X_{k}^{k, u_{k}, \bar{u}_{k}, \Phi, \lambda}= & X_{k}^{t, x, *}
\end{aligned}\right.
$$

Noting that $X_{k}^{k, u_{k}, \Phi}=X_{k}^{k, u_{k}, \bar{u}_{k}, \Phi, \lambda}=X_{k}^{t, x, *}$, we have

$$
\left\{\begin{aligned}
\frac{X_{\ell+1}^{k, u_{k}, \bar{u}_{k}, \Phi, \lambda}-X_{\ell+1}^{k, u_{k}, \Phi}}{\lambda}= & \left(A_{\ell}+B_{\ell} \Phi_{\ell}\right) \frac{X_{\ell}^{k, u_{k}, \bar{u}_{k}, \Phi, \lambda}-X_{\ell}^{k, u_{k}, \Phi}}{\lambda} \\
& +\left(C_{\ell}+D_{\ell} \Phi_{\ell}\right) \frac{X_{\ell}^{k, u_{k}, \bar{u}_{k}, \Phi, \lambda}-X_{\ell}^{k, u_{k}, \Phi}}{\lambda} w_{\ell}, \\
\frac{X_{k+1}^{k, u_{k}, \bar{u}_{k}, \Phi, \lambda}-X_{k+1}^{k, u_{k}, \Phi}}{\lambda}= & {\left[A_{k} \frac{X_{k}^{k, u_{k}, \bar{u}_{k}, \Phi, \lambda}-X_{k}^{k, u_{k}, \Phi}}{\lambda}+B_{k} \bar{u}_{k}\right] } \\
& +\left[C_{k} \frac{X_{k}^{k, u_{k}, \bar{u}_{k}, \Phi, \lambda}-X_{k}^{k, u_{k}, \Phi}}{\lambda}+D_{k} \bar{u}_{k}\right] w_{k}, \\
\frac{X_{k}^{k, u_{k}, \bar{u}_{k}, \Phi, \lambda}-X_{k}^{k, u_{k}, \Phi}}{\lambda}= & 0, \quad \ell \in \mathbb{T}_{k+1} .
\end{aligned}\right.
$$

Denote $\frac{X_{\ell}^{k, u_{k}, \bar{u}_{k}, \Phi, \lambda}-X_{\ell}^{k, u_{k}, \Phi}}{\lambda}$ by $Y_{\ell}^{k, \bar{u}_{k}, \Phi}$, which is independent of $u_{k}$ and $\lambda$. Then, we can get (36), which implies

$$
\left\{\begin{array}{l}
\mathbb{E}_{k} Y_{\ell+1}^{k, \bar{u}_{k}, \Phi}=\left(A_{\ell}+B_{\ell} \Phi_{\ell}^{t, x}\right) \mathbb{E}_{k} Y_{\ell}^{k, \bar{u}_{k}, \Phi} \\
\mathbb{E}_{k} Y_{k+1}^{k, \bar{u}_{k}, \Phi}=B_{k} \bar{u}_{k} \\
\mathbb{E}_{k} Y_{k}^{k, \bar{u}_{k}, \Phi}=0
\end{array}\right.
$$

and

$$
\left\{\begin{aligned}
Y_{\ell+1}^{k, \bar{u}_{k}, \Phi}-\mathbb{E}_{k} Y_{\ell+1}^{k, \bar{u}_{k}, \Phi}= & \left(A_{\ell}+B_{\ell} \Phi_{\ell}\right)\left(Y_{\ell}^{k, \bar{u}_{k}, \Phi}-\mathbb{E}_{k} Y_{\ell}^{k, \bar{u}_{k}, \Phi}\right) \\
& +\left[\left(C_{\ell}+D_{\ell} \Phi_{\ell}\right)\left(Y_{\ell}^{k, \bar{u}_{k}, \Phi}-\mathbb{E}_{k} Y_{\ell}^{k, \bar{u}_{k}, \Phi}\right)\right. \\
& \left.+\left(C_{\ell}+D_{\ell} \Phi_{\ell}\right) \mathbb{E}_{k} Y_{\ell}^{k, \bar{u}_{k}, \Phi}\right] w_{\ell}, \\
Y_{k+1}^{k, \bar{u}_{k}, \Phi}-\mathbb{E}_{k} Y_{k+1}^{k, \bar{u}_{k}, \Phi}= & D_{k} \bar{u}_{k} w_{k}, \\
Y_{k}^{k, \bar{u}_{k}, \Phi}-\mathbb{E}_{k} Y_{k}^{k, \bar{u}_{k}, \Phi}= & 0, \quad \ell \in \mathbb{N}_{k+1} .
\end{aligned}\right.
$$

Noting

$$
X_{\ell}^{k, u_{k}, \bar{u}_{k}, \Phi, \lambda}=X_{\ell}^{k, u_{k}, \Phi}+\lambda Y_{\ell}^{k, \bar{u}_{k}, \Phi}, \quad \ell \in \mathbb{T}_{k},
$$


we have similarly to (12)

$$
\begin{aligned}
\text { (40) } & d \widetilde{J}\left(k, X_{k}^{t, x, *} ; u_{k} ; \bar{u}_{k}\right)=\lim _{\lambda \downarrow 0} \frac{\widetilde{J}\left(k, X_{k}^{t, x, *} ; u_{k}+\lambda \bar{u}_{k}\right)-\widetilde{J}\left(k, X_{k}^{t, x, *} ; u_{k}\right)}{\lambda} \\
= & 2\left\langle Q X^{k, u_{k}, \Phi}, Y^{k, \bar{u}_{k}, \Phi}\right\rangle_{\mathbb{T}_{k}}+2\left\langle\bar{Q} \mathbb{E}_{k} X^{k, u_{k}, \Phi}, \mathbb{E}_{k} Y^{k, \bar{u}_{k}, \Phi}\right\rangle_{\mathbb{T}_{k}}+2\left\langle R_{k} u_{k}, \bar{u}_{k}\right\rangle_{k} \\
& +2\left\langle R \Phi X^{k, u_{k}, \Phi}, \Phi Y^{k, \bar{u}_{k}, \Phi}\right\rangle_{\mathbb{T}_{k+1}}+2\left\langle\bar{R}_{k} \mathbb{E}_{k} u_{k}, \mathbb{E}_{k} \bar{u}_{k}\right\rangle_{k} \\
& +2\left\langle\bar{R} \mathbb{E}_{k}\left(\Phi X^{k, u_{k}, \Phi}\right), \mathbb{E}_{k}\left(\Phi Y^{k, \bar{u}_{k}, \Phi}\right)\right\rangle_{\mathbb{T}_{k+1}}+2\left\langle G X_{N}^{k, u_{k}, \Phi}, Y_{N}^{k, \bar{u}_{k}, \Phi}\right\rangle_{N} \\
& +2\left\langle\bar{G} \mathbb{E}_{k} X_{N}^{k, u_{k}, \Phi}, \mathbb{E}_{k} Y_{N}^{k, \bar{u}_{k}, \Phi}\right\rangle_{N},
\end{aligned}
$$

and

$$
\begin{aligned}
& d^{2} \widetilde{J}\left(k, X_{k}^{t, x, *} ; u_{k} ; \bar{u}_{k} ; \hat{u}_{k}\right) \\
= & \lim _{\lambda \downarrow 0} \frac{d \widetilde{J}\left(k, X_{k}^{t, x, *} ; u_{k}+\beta \hat{u}_{k} ; \bar{u}_{k}\right)-d \widetilde{J}\left(k, X_{k}^{t, x, *} ; u_{k} ; \bar{u}_{k}\right)}{\beta} \\
= & 2\left\langle Q Y^{k, \hat{u}_{k}, \Phi}, Y^{k, \bar{u}_{k}, \Phi}\right\rangle_{\mathbb{T}_{k}}+2\left\langle\bar{Q} \mathbb{E}_{k} Y^{k, \hat{u}_{k}, \Phi}, \mathbb{E}_{k} Y^{k, \bar{u}_{k}, \Phi}\right\rangle_{\mathbb{T}_{k}}+2\left\langle R_{k} \hat{u}_{k}, \bar{u}_{k}\right\rangle_{k} \\
& +2\left\langle R \Phi Y^{k, \hat{u}_{k}, \Phi}, \Phi^{t, x} Y^{k, \bar{u}_{k}, \Phi}\right\rangle_{\mathbb{T}_{k+1}}+2\left\langle\bar{R}_{k} \mathbb{E}_{k} \hat{u}_{k}, \mathbb{E}_{k} \bar{u}_{k}\right\rangle_{k} \\
& +2\left\langle\bar{R} \mathbb{E}_{k}\left(\Phi Y^{k, \hat{u}_{k}, \Phi}\right), \mathbb{E}_{k}\left(\Phi^{t, x} Y^{k, \bar{u}_{k}, \Phi}\right)\right\rangle_{\mathbb{T}_{k+1}}+2\left\langle G Y_{N}^{k, \hat{u}_{k}, \Phi}, Y_{N}^{k, \bar{u}_{k}, \Phi}\right\rangle_{N} \\
& +2\left\langle\bar{G} \mathbb{E}_{k} Y_{N}^{k, \hat{u}_{k}, \Phi}, \mathbb{E}_{k} Y_{N}^{k, \bar{u}_{k}, \Phi}\right\rangle_{N}
\end{aligned}
$$

with $u_{k}, \bar{u}_{k}, \hat{u}_{k} \in L_{\mathcal{F}}^{2}\left(k ; \mathbb{R}^{m}\right)$, and $Y^{k, \hat{u}_{k}, \Phi}$ being similarly defined as (36). If $\hat{u}_{k}=\bar{u}_{k}$, then it holds that

$$
\begin{aligned}
& d^{2} \widetilde{J}\left(k, X_{k}^{t, x, *} ; u_{k} ; \bar{u}_{k} ; \bar{u}_{k}\right) \\
= & 2\left\langle Q Y^{k, \bar{u}_{k}, \Phi}, Y^{k, \bar{u}_{k}, \Phi}\right\rangle_{\mathbb{T}_{k}}+2\left\langle\bar{Q} \mathbb{E}_{k} Y^{k, \bar{u}_{k}, \Phi}, \mathbb{E}_{k} Y^{k, \bar{u}_{k}, \Phi}\right\rangle_{\mathbb{T}_{k}}+2\left\langle R_{k} \bar{u}_{k}, \bar{u}_{k}\right\rangle_{k} \\
& +2\left\langle R \Phi Y^{k, \bar{u}_{k}, \Phi}, \Phi^{t, x} Y^{k, \bar{u}_{k}, \Phi}\right\rangle_{\mathbb{T}_{k+1}}+2\left\langle\bar{R}_{k} \mathbb{E}_{k} \bar{u}_{k}, \mathbb{E}_{k} \bar{u}_{k}\right\rangle_{k} \\
& +2\left\langle\bar{R} \mathbb{E}_{k}\left(\Phi Y^{k, \bar{u}_{k}, \Phi}\right), \mathbb{E}_{k}\left(\Phi Y^{k, \bar{u}_{k}, \Phi}\right)\right\rangle_{\mathbb{T}_{k+1}}+2\left\langle G Y_{N}^{k, \bar{u}_{k}, \Phi}, Y_{N}^{k, \bar{u}_{k}, \Phi}\right\rangle_{N} \\
& +2\left\langle\bar{G}_{k} \mathbb{E} Y_{N}^{k, \hat{u}_{k}, \Phi}, \mathbb{E}_{k} Y_{N}^{k, \bar{u}_{k}, \Phi}\right\rangle_{N} .
\end{aligned}
$$

Letting $u_{k}=\Phi_{k} X_{k}^{t, x, *}$ in (40), we have

$$
\begin{aligned}
& \frac{1}{2} d \widetilde{J}\left(k, X_{k}^{t, x, *} ; \Phi_{k} X_{k}^{t, x, *} ; \bar{u}_{k}\right) \\
= & \left\langle Q X^{k, \Phi}, Y^{k, \bar{u}_{k}, \Phi}\right\rangle_{\mathbb{T}_{k}}+\left\langle\bar{Q} \mathbb{E}_{k} X^{k, \Phi}, \mathbb{E}_{k} Y^{k, \bar{u}_{k}, \Phi}\right\rangle_{\mathbb{T}_{k}}+\left\langle R_{k} \Phi_{k}^{t, x} X_{k}^{t, x, *}, \bar{u}_{k}\right\rangle_{k} \\
& +\left\langle R \Phi X^{k, \Phi}, \Phi^{t, x} Y^{k, \bar{u}_{k}, \Phi}\right\rangle_{\mathbb{T}_{k+1}}+\left\langle\bar{R}_{k} \mathbb{E}_{k}\left(\Phi_{k} X_{k}^{t, x, *}\right), \mathbb{E}_{k} \bar{u}_{k}\right\rangle_{k}
\end{aligned}
$$




$$
\begin{aligned}
& +\left\langle\bar{R}\left(\mathbb{E}_{k}\left(\Phi X^{k, \Phi}\right), \mathbb{E}_{k}\left(\Phi Y^{k, \bar{u}_{k}, \Phi}\right)\right\rangle_{\mathbb{T}_{k+1}}+\left\langle G X_{N}^{k, \Phi}, Y_{N}^{k, \bar{u}_{k}, \Phi}\right\rangle_{N}\right. \\
& +\left\langle\bar{G} \mathbb{E}_{k} X_{N}^{k, \Phi}, \mathbb{E}_{k} Y_{N}^{k, \bar{u}_{k}, \Phi}\right\rangle_{N} \\
& =\sum_{\ell=k}^{N-1} \mathbb{E}_{k}\left[\left(Q_{\ell}\left(X_{\ell}^{k, \Phi}-\mathbb{E}_{k} X_{\ell}^{k, \Phi}\right)+\left(\Phi_{\ell}\right)^{T} R_{\ell} \Phi_{\ell}\left(X_{\ell}^{k, \Phi}-X_{\ell}^{k, \Phi} \mathbb{E}_{k}\right)\right.\right. \\
& \left.+\left(\mathcal{Q}_{\ell} \mathbb{E}_{k} X_{\ell}^{k, \Phi}+\left(\Phi_{\ell}\right)^{T} \mathcal{R}_{\ell} \Phi_{\ell} \mathbb{E}_{k} X_{\ell}^{k, \Phi}\right)^{T} \mathbb{E}_{k} Y_{\ell}^{k, \bar{u}_{k}, \Phi}\right] \\
& +\left[\mathcal{R}_{k} \Phi_{k} X_{k}^{t, x, *}\right]^{T} \bar{u}_{k} \\
& +\mathbb{E}_{k}\left[\left(G\left(X_{N}^{k, \Phi}-\mathbb{E}_{k} X_{N}^{k, \Phi}\right)\right)^{T}\left(Y_{N}^{k, \bar{u}_{k}, \Phi}-\mathbb{E}_{k} Y_{N}^{k, \bar{u}_{k}, \Phi}\right)\right] \\
& +\left[\left(\mathcal{G} \mathbb{E}_{k} X_{N}^{k, \Phi}\right)^{T} \mathbb{E}_{k} Y_{N}^{k, \bar{u}_{k}, \Phi}\right] \\
& =\sum_{\ell=k}^{N-1} \mathbb{E}_{k}\left\{\left[Q_{\ell}\left(X_{\ell}^{k, \Phi}-\mathbb{E}_{k} X_{\ell}^{k, \Phi}\right)+\Phi_{\ell}^{T} R_{\ell} \Phi_{\ell}^{t, x}\left(X_{\ell}^{k, \Phi}-\mathbb{E}_{k} X_{\ell}^{k, \Phi}\right)\right.\right. \\
& +\left(A_{\ell}+B_{\ell} \Phi_{\ell}\right)^{T}\left(Z_{\ell+1}^{k, \Phi}-\mathbb{E}_{k} Z_{\ell+1}^{k, \Phi}\right) \\
& +\left(C_{\ell}+D_{\ell} \Phi_{\ell}\right)^{T}\left(\mathbb{E}_{\ell}\left(Z_{\ell+1}^{k, \Phi} w_{\ell}\right)-\mathbb{E}_{k}\left(Z_{\ell+1}^{k, \Phi} w_{\ell}\right)\right) \\
& \left.-\left(Z_{\ell}^{k, \Phi}-Z_{\ell}^{k, \Phi} \mathbb{E}_{k}\right)\right]^{T}\left(Y_{\ell}^{k, \bar{u}_{k}, \Phi}-\mathbb{E}_{k} Y_{\ell}^{k, \bar{u}_{k}, \Phi}\right) \\
& +\left[\mathcal{Q}_{\ell} \mathbb{E}_{k} X_{\ell}^{k, \Phi}+\Phi_{\ell}^{T} \mathcal{R}_{\ell} \Phi_{\ell} \mathbb{E}_{k} X_{\ell}^{k, \Phi}+\left(A_{\ell}+B_{\ell} \Phi_{\ell}\right)^{T} \mathbb{E}_{k} Z_{\ell+1}^{k, \Phi}\right. \\
& \left.\left.+\left(C_{\ell}+D_{\ell} \Phi_{\ell}^{t, x}\right)^{T} \mathbb{E}_{k}\left(Z_{\ell+1}^{k, \Phi} w_{\ell}\right)-\mathbb{E}_{k} Z_{\ell}^{k, \Phi}\right]^{T} \mathbb{E}_{k} Y_{\ell}^{k, \bar{u}_{k}, \Phi}\right\} \\
& +\left[\mathcal{R}_{k} \Phi_{k}^{t, x} X_{k}^{t, x, *}+B_{k}^{T} \mathbb{E}_{k} Z_{k+1}^{k, \Phi}+D_{k}^{T} \mathbb{E}_{k}\left(Z_{k+1}^{k, \Phi} w_{k}\right)\right]^{T} \bar{u}_{k} .
\end{aligned}
$$

Based on this and the proof of Theorem 3, we can achieve the desired result of Theorem 7 .

From above theorem, we can derive a result which characterizes the existence of the closed-loop equilibrium strategy via the solvability of two coupled equations.

Theorem 8. The following statements are equivalent.

(i). There exists a closed-loop equilibrium strategy of Problem (LQ). 
(ii). The following coupled difference equations

$$
\left\{\begin{aligned}
& S_{k}= Q_{k}+\Phi_{k}^{T} R_{k} \Phi_{k}+\left(A_{k}+B_{k} \Phi_{k}\right)^{T} S_{k+1}\left(A_{k}+B_{k} \Phi_{k}\right) \\
&+\left(C_{k}+D_{k} \Phi_{k}\right)^{T} S_{k+1}\left(C_{k}+D_{k} \Phi_{k}\right) \\
& \bar{S}_{k}=\bar{Q}_{k}+\Phi_{k}^{T} \bar{R}_{k} \Phi_{k}+\left(A_{k}+B_{k} \Phi_{k}\right)^{T} \bar{S}_{k+1}\left(A_{k}+B_{k} \Phi_{k}\right) \\
& S_{N}=G, \quad \bar{S}_{N}=\bar{G} \\
& \mathcal{O}_{k} \geq 0 \\
& \mathcal{O}_{k} \mathcal{O}_{k}^{\dagger} \mathcal{L}_{k}-\mathcal{L}_{k}=0 \\
& k \in \mathbb{T}
\end{aligned}\right.
$$

are solvable in the sense that $\mathcal{O}_{k} \geq 0, \mathcal{O}_{k, k} \mathcal{O}_{k, k}^{\dagger} \mathcal{L}_{k, k}-\mathcal{L}_{k, k}=0, k \in \mathbb{T}$, hold. In (42), $\mathcal{O}_{k}, \mathcal{L}_{k}$ and $\Phi_{k}$ are given by

$$
\left\{\begin{array}{l}
\mathcal{O}_{k}=\mathcal{R}_{k}+B_{k}^{T}\left(S_{k+1}+\bar{S}_{k+1}\right) B_{k}+D_{k}^{T} S_{k+1} \mathcal{D}_{k} \\
\mathcal{L}_{k}=B_{k}^{T}\left(S_{k+1}+\bar{S}_{k+1}\right) A_{k}+D_{k}^{T} S_{k+1} C_{k} \\
\Phi_{k}=-\mathcal{O}_{k}^{\dagger} \mathcal{L}_{k}
\end{array}\right.
$$

In this case, a closed-loop equilibrium strategy is given by

$$
\Phi_{k}=-\mathcal{O}_{k}^{\dagger} \mathcal{L}_{k}, \quad k \in \mathbb{T}
$$

Proof. (i) $\Rightarrow$ (ii). By Theorem 7, we have the solvability of (32) with properties (33) and (34). We now characterize $\Phi$. Noting that $Z_{N}^{k, \Phi}=G X_{N}^{k, \Phi}+$ $\bar{G}_{k} \mathbb{E}_{k} X_{N}^{k, \Phi}$, one can get

$$
\begin{aligned}
& \left(A_{N-1}+B_{N-1} \Phi_{N-1}\right)^{T} \mathbb{E}_{N-1} Z_{N}^{k, \Phi} \\
= & \left(A_{N-1}+B_{N-1} \Phi_{N-1}\right)^{T} G\left(A_{N-1}+B_{N-1} \Phi_{N-1}\right) X_{N-1}^{k, \Phi} \\
& +\left(A_{N-1}+B_{N-1} \Phi_{N-1}\right)^{T}\left[G\left(\bar{A}_{N-1}+\bar{B}_{N-1} \Phi_{N-1}\right)\right. \\
& \left.+\bar{G}\left(\mathcal{A}_{N-1}+\mathcal{B}_{N-1} \Phi_{N-1}\right)\right] \mathbb{E}_{k} X_{N-1}^{k, \Phi},
\end{aligned}
$$

and

$$
\begin{aligned}
& \left(C_{N-1}+D_{N-1} \Phi_{N-1}\right)^{T} \mathbb{E}_{N-1}\left(Z_{N}^{k, \Phi} w_{N-1}\right) \\
= & \left(C_{N-1}+D_{N-1} \Phi_{N-1}\right)^{T} G\left(C_{N-1}+D_{N-1} \Phi_{N-1}\right) X_{N-1}^{k, \Phi}
\end{aligned}
$$




$$
+\left(C_{N-1}+D_{N-1} \Phi_{N-1}\right)^{T} G\left(\bar{C}_{N-1}+\bar{D}_{N-1} \Phi_{N-1}\right) \mathbb{E}_{k} X_{N-1}^{k, \Phi}
$$

Therefore, from the expression of $Z^{k, \Phi}$ we have

$$
\begin{aligned}
Z_{N-1}^{k, \Phi}= & {\left[Q_{N-1}+\Phi_{N-1}^{T} R_{N-1} \Phi_{N-1}\right.} \\
& +\left(A_{N-1}+B_{N-1} \Phi_{N-1}\right)^{T} G\left(A_{N-1}+B_{N-1} \Phi_{N-1}\right) \\
& \left.+\left(C_{N-1}+D_{N-1} \Phi_{N-1}\right)^{T} G\left(C_{N-1}+D_{N-1} \Phi_{N-1}\right)\right] X_{N-1}^{k, \Phi} \\
& +\left[\bar{Q}_{N-1}+\Phi_{N-1}^{T} \bar{R}_{N-1} \Phi_{N-1}\right. \\
& \left.+\left(A_{N-1}+B_{N-1} \Phi_{N-1}\right)^{T} \bar{G}\left(A_{N-1}+B_{N-1} \Phi_{N-1}\right)\right] \mathbb{E}_{k} X_{N-1}^{k, \Phi} \\
= & Q_{N-1}+\Phi_{N-1}^{T} R_{N-1} \Phi_{N-1} \\
& +\left(A_{N-1}+B_{N-1} \Phi_{N-1}\right)^{T} G\left(A_{N-1}+B_{N-1} \Phi_{N-1}\right) \\
& \left.+\left(C_{N-1}+D_{N-1} \Phi_{N-1}\right)^{T} G\left(C_{N-1}+D_{N-1} \Phi_{N-1}\right)\right] \\
& \times\left(X_{N-1}^{k, \Phi}-\mathbb{E}_{k} X_{N-1}^{k, \Phi}\right) \\
& +\left[\mathcal{Q}_{N-1}+\Phi_{N-1}^{T} \mathcal{R}_{N-1} \Phi_{N-1}\right. \\
& +\left(A_{N-1}+B_{N-1} \Phi_{N-1}\right)^{T} \mathcal{G}\left(A_{N-1}+B_{N-1} \Phi_{N-1}\right) \\
& \left.+\left(C_{N-1}+D_{N-1} \Phi_{N-1}\right)^{T} G\left(C_{N-1}+D_{N-1} \Phi_{N-1}\right)\right] \mathbb{E}_{k} X_{N-1}^{k, \Phi}
\end{aligned}
$$

Furthermore, we have

$$
B_{N-1}^{T} \mathbb{E}_{N-1} Z_{N}^{N-1, \Phi}=B_{N-1}^{T}(G+\bar{G})\left(A_{N-1}+B_{N-1} \Phi_{N-1}\right) X_{N-1}^{N-1, \Phi},
$$

and

$$
D_{N-1}^{T} \mathbb{E}_{N-1}\left(Z_{N}^{N-1, \Phi} w_{N-1}\right)=D_{N-1}^{T} G\left(C_{N-1}+D_{N-1} \Phi_{N-1}\right) X_{N-1}^{N-1, \Phi}
$$

which together with (33) imply

$$
\begin{aligned}
0= & {\left[\left(\mathcal{R}_{N-1}+B_{N-1}^{T}(G+\bar{G}) B_{N-1}+D_{N-1}^{T} G D_{N-1}\right) \Phi_{N-1}\right.} \\
& \left.+B_{N-1}^{T}(G+\bar{G}) A_{N-1}+D_{N-1}^{T} G C_{N-1}\right] X_{N-1}^{N-1, \Phi}
\end{aligned}
$$


Concerned with Problem (LQ) for the initial pair $(N-1, x)$ and $x \in$ $L_{\mathcal{F}}^{2}\left(N-1 ; \mathbb{R}^{n}\right)$, we have

$$
\begin{aligned}
0= & \left(\mathcal{R}_{N-1}+B_{N-1}^{T} G B_{N-1}+D_{N-1}^{T} G D_{N-1}\right) \Phi_{N-1} \\
& +B_{N-1}^{T} G_{k} A_{N-1}+D_{N-1}^{T} G C_{N-1},
\end{aligned}
$$

as $x$ can be arbitrarily selected. From Lemma 4 , it follows that

$$
\Phi_{N-1}=-\mathcal{O}_{N-1}^{\dagger} \mathcal{L}_{N-1}
$$

and

$$
\mathcal{O}_{N-1} \mathcal{O}_{N-1}^{\dagger} \mathcal{L}_{N-1}-\mathcal{L}_{N-1}=0
$$

where

$$
\left\{\begin{array}{l}
\mathcal{O}_{N-1}=\mathcal{R}_{N-1}+B_{N-1}^{T}(G+\bar{G}) B_{N-1}+D_{N-1}^{T} G D_{N-1} \\
\mathcal{L}_{N-1}=B_{N-1}^{T}(G+\bar{G}) A_{N-1}+D_{N-1}^{T} G C_{N-1}
\end{array}\right.
$$

Let

$$
\left\{\begin{aligned}
S_{N-1}= & Q_{N-1}+\Phi_{N-1}^{T} R_{N-1} \Phi_{N-1} \\
& +\left(A_{N-1}+B_{N-1} \Phi_{N-1}\right)^{T} G\left(A_{N-1}+B_{N-1} \Phi_{N-1}\right) \\
& +\left(C_{N-1}+D_{N-1} \Phi_{N-1}\right)^{T} G\left(C_{N-1}+D_{N-1} \Phi_{N-1}\right) \\
\bar{S}_{k, N-1}= & \bar{Q}_{N-1}+\Phi_{N-1}^{T} \bar{R}_{N-1} \Phi_{N-1} \\
& +\left(A_{N-1}+B_{N-1} \Phi_{N-1}\right)^{T} \bar{G}\left(A_{N-1}+B_{N-1} \Phi_{N-1}\right) .
\end{aligned}\right.
$$

Then, by (44) we have

$$
Z_{N-1}^{k, \Phi}=S_{N-1} X_{N-1}^{k, \Phi}+\bar{S}_{N-1} \mathbb{E}_{k} X_{N-1}^{k, \Phi}
$$

Repeating the analysis between (44) and (45), we can get the results except for $\mathcal{O}_{k} \geq 0, k \in \mathbb{T}$.

To this end, we prove $\mathcal{O}_{k} \geq 0, k \in \mathbb{T}$. From (34), we have for any $\bar{u}_{k} \in$ $L_{\mathcal{F}}^{2}\left(k ; \mathbb{R}^{m}\right)$ that

$$
\begin{aligned}
0 \leq & \left\langle Q Y^{k, \bar{u}_{k}, \Phi}, Y^{k, \bar{u}_{k}, \Phi}\right\rangle_{\mathbb{T}_{k}}+\left\langle\bar{Q} \mathbb{E}_{k} Y^{k, \bar{u}_{k}, \Phi}, \mathbb{E}_{k} Y^{k, \bar{u}_{k}, \Phi}\right\rangle_{\mathbb{T}_{k}}+\left\langle R_{k} \bar{u}_{k}, \bar{u}_{k}\right\rangle_{k} \\
& +\left\langle R \Phi Y^{k, \bar{u}_{k}, \Phi}, \Phi Y^{k, \bar{u}_{k}, \Phi}\right\rangle_{\mathbb{T}_{k+1}}+\left\langle\bar{R}_{k} \mathbb{E}_{k} \bar{u}_{k}, \mathbb{E}_{k} \bar{u}_{k}\right\rangle_{k} \\
& +\left\langle\bar{R} \mathbb{E}_{k}\left(\Phi Y^{k, \bar{u}_{k}, \Phi}\right), \mathbb{E}_{k}\left(\Phi Y^{k, \bar{u}_{k}, \Phi}\right)\right\rangle_{\mathbb{T}_{k+1}}+\left\langle G Y_{N}^{k, \bar{u}_{k}, \Phi}, Y_{N}^{k, \bar{u}_{k}, \Phi}\right\rangle_{N}
\end{aligned}
$$




$$
\begin{aligned}
& +\left\langle\bar{G} \mathbb{E}_{k} Y_{N}^{k, \bar{u}_{k}, \Phi}, \mathbb{E}_{k} Y_{N}^{k, \bar{k}_{k}, \Phi}\right\rangle_{N} \\
= & \sum_{\ell=k}^{N-1}\left\{\mathbb { E } _ { k } \left[Y _ { \ell } ^ { k , \overline { u } _ { k } , \Phi } \left(Q_{\ell}+\Phi_{\ell}^{T} R_{\ell} \Phi_{\ell}+\left(A_{\ell}+B_{\ell} \Phi_{\ell}\right)^{T} S_{\ell+1}\left(A_{\ell}+B_{\ell} \Phi_{\ell}\right)\right.\right.\right. \\
& \left.\left.+\left(C_{\ell}+D_{\ell} \Phi_{\ell}\right)^{T} S_{\ell+1}\left(C_{\ell}+D_{\ell} \Phi_{\ell}\right)-S_{\ell}\right) Y_{\ell}^{k, \bar{u}_{k}, \Phi}\right] \\
& +\left(\mathbb{E}_{k} Y_{\ell}^{k, \bar{u}_{k}, \Phi}\right)^{T}\left(\mathcal{Q}_{\ell}+\Phi_{\ell}^{T} \mathcal{R}_{\ell} \Phi_{\ell}\right. \\
& +\left(A_{\ell}+B_{\ell} \Phi_{\ell}\right)^{T}\left(S_{\ell+1}+\bar{S}_{\ell+1}\right)\left(A_{\ell}+B_{\ell} \Phi_{\ell}\right) \\
& \left.\left.+\left(C_{\ell}+D_{\ell} \Phi_{\ell}\right)^{T} S_{\ell+1}\left(C_{\ell}+D_{\ell} \Phi_{\ell}\right)-\left(S_{\ell}+\bar{S}_{\ell}\right)\right) \mathbb{E}_{k} Y_{\ell}^{k, \bar{u}_{k}, \Phi}\right\} \\
& +\bar{u}_{k}^{T}\left[\mathcal{R}_{k}+B_{k}^{T}\left(S_{k+1}+\bar{S}_{k+1}\right) B_{k}+D_{k}^{T} S_{k+1} D_{k}\right] \bar{u}_{k} \\
= & \bar{u}_{k}^{T}\left[\mathcal{R}_{k}+B_{k}^{T}\left(S_{k+1}+\bar{S}_{k+1}\right) B_{k}+D_{k}^{T} S_{k+1} D_{k}\right] \bar{u}_{k} \\
= & \bar{u}_{k}^{T} \mathcal{O}_{k} \bar{u}_{k} .
\end{aligned}
$$

Hence, $\mathcal{O}_{k} \geq 0$ for any $k \in \mathbb{T}$.

(ii) $\Rightarrow(\mathrm{i})$. By reversing above procedure, we can get the results.

If (42) is solvable, then we have $S_{k}^{T}=S_{k}, \bar{S}_{k}=\bar{S}_{k}, k \in \mathbb{T}$. In this case, we call (42) has the symmetric structure. Instead, the GDREs (22) for the open-loop formulation do not have symmetric structure. Let $\mathcal{S}_{k}=S_{k}+\bar{S}_{k}$, $k \in \mathbb{T}$ and $\mathcal{G}=G+\bar{G}$. Then, (42) can be rewritten as

$$
\left\{\begin{aligned}
& S_{k}= Q_{k}+A_{k}^{T} S_{k+1} A_{k}+C_{k}^{T} S_{k+1} C_{k}-L_{k}^{T} \mathcal{O}_{k}^{\dagger} \mathcal{L}_{k} \\
& \quad-\mathcal{L}_{k}^{T} \mathcal{O}_{k}^{\dagger} L_{k}+\mathcal{L}_{k}^{T} \mathcal{O}_{k}^{\dagger} O_{k} \mathcal{O}_{k}^{\dagger} \mathcal{L}_{k} \\
& \mathcal{S}_{k}= \mathcal{Q}_{k}+A_{k}^{T} \mathcal{S}_{k+1} A_{k}+C_{k}^{T} S_{k+1} C_{k}-\mathcal{L}_{k}^{T} \mathcal{O}_{k}^{\dagger} \mathcal{L}_{k} \\
& S_{N}=G, \quad \mathcal{S}_{N}=\mathcal{G} \\
& \mathcal{O}_{k} \geq 0 \\
& \mathcal{O}_{k} \mathcal{O}_{k}^{\dagger} \mathcal{L}_{k}-\mathcal{L}_{k}=0 \\
& k \in \mathbb{T}
\end{aligned}\right.
$$

where

$$
\left\{\begin{array}{l}
O_{k}=R_{k}+B_{k}^{T} S_{k+1} B_{k}+D_{k}^{T} S_{k+1} \mathcal{D}_{k}, \\
L_{k}=B_{k}^{T} S_{k+1} A_{k}+D_{k}^{T} S_{k+1} C_{k}, \\
\mathcal{O}_{k}=\mathcal{R}_{k}+B_{k}^{T} \mathcal{S}_{k+1} B_{k}+D_{k}^{T} S_{k+1} \mathcal{D}_{k}, \\
\mathcal{L}_{k}=B_{k}^{T} \mathcal{S}_{k+1} A_{k}+D_{k}^{T} S_{k+1} C_{k}, \\
k \in \mathbb{T}
\end{array}\right.
$$


Let us review a static mean-field stochastic LQ problem, denoted as Problem (sLQ), whose system equation is (1) and the cost functional is

$$
\begin{aligned}
J(t, x ; u)= & \sum_{k=t}^{N-1} \mathbb{E}\left[\left(X_{k}^{t}\right)^{T} Q_{k} X_{k}^{t}+\left(\mathbb{E} X_{k}^{t}\right)^{T} \bar{Q}_{k} \mathbb{E} X_{k}^{t}+u_{k}^{T} R_{k} u_{k}\right. \\
& \left.+\left(\mathbb{E} u_{k}\right)^{T} \bar{R}_{k} \mathbb{E} u_{k}\right]+\mathbb{E}\left[\left(X_{N}^{t}\right)^{T} G X_{N}^{t}\right]+\left(\mathbb{E} X_{N}^{t}\right)^{T} \bar{G} \mathbb{E} X_{N}^{t}
\end{aligned}
$$

So, the expectations in (47) are different from those in (2) that are the conditional expectations. This is why we call Problem (sLQ) a static problem. From [9], the existence of optimal control of Problem (sLQ) for all the initial pairs is equivalent to the existence of the closed-loop optimal strategy and is also equivalent to the solvability of the following equations

$$
\left\{\begin{array}{l}
P_{k}=Q_{k}+A_{k}^{\tau} P_{k+1} A_{k}+C_{k}^{T} P_{k+1} C_{k}-H_{k}^{T} W_{k}^{\dagger} H_{k} \\
\mathcal{P}_{k}=\mathcal{Q}_{k}+A_{k}^{T} \mathcal{P}_{k+1} A_{k}+C_{k}^{T} P_{k+1} C_{k}-\mathcal{H}_{k}^{T} \mathcal{W}_{k}^{\dagger} \mathcal{H}_{k} \\
P_{N}=G_{N}, \quad \mathcal{P}_{N}=G+\bar{G} \\
W_{k}, \mathcal{W}_{k} \geq 0, \quad W_{k} W_{k}^{\dagger} H_{k}-H_{k}=0 \\
\mathcal{W}_{k} \mathcal{W}_{k}^{T} \mathcal{H}_{k}-\mathcal{H}_{k}=0 \\
k \in \mathbb{T}
\end{array}\right.
$$

where

$$
\left\{\begin{array}{l}
W_{k}=R_{k}+B_{k}^{\tau} P_{k+1} B_{k}+D_{k}^{\tau} P_{k+1} D_{k} \\
H_{k}=B_{k}^{\tau} P_{k+1} A_{k}+D_{k}^{\tau} P_{k+1} C_{k} \\
\mathcal{W}_{k}=\mathcal{R}_{k}+B_{k}^{T} \mathcal{P}_{k+1} B_{k}+D_{k}^{T} P_{k+1} D_{k} \\
\mathcal{H}_{k}=B_{k}^{T} \mathcal{P}_{k+1} A_{k}+D_{k}^{T} P_{k+1} C_{k} \\
k \in \mathbb{T}
\end{array}\right.
$$

Here, (48) is called solvable if $W_{k}, \mathcal{W}_{k} \geq 0, W_{k} W_{k}^{\dagger} H_{k}-H_{k}=0, \mathcal{W}_{k} \mathcal{W}_{k}^{T} \mathcal{H}_{k}-$ $\mathcal{H}_{k}=0, k \in \mathbb{T}$, are satisfied. Note that the GDREs (48) differs (46) and (21)(22). This reveals the essential difference between the static mean-field LQ problem (Problem (sLQ)) and the dynamic mean-field LQ problem (Problem $(\mathrm{LQ}))$.

\section{Example}

In this section, an example is presented to illustrate the theory developed in above sections. 
Example 1. Consider a Problem (LQ), whose system matrices and weighting matrices are given below

$$
\begin{aligned}
& A_{0}=\left[\begin{array}{cc}
2.3 & 1.41 \\
-0.83 & 2.59
\end{array}\right], \quad A_{1}=\left[\begin{array}{cc}
2.32 & -1.35 \\
-1.43 & 3.78
\end{array}\right] \text {, } \\
& A_{2}=\left[\begin{array}{cc}
1.34 & -1.32 \\
0.43 & 1.73
\end{array}\right], \quad B_{0}=\left[\begin{array}{cc}
3.15 & -1.3 \\
2.2 & 4.01
\end{array}\right] \text {, } \\
& B_{1}=\left[\begin{array}{ll}
-3.45 & -1.06 \\
-0.52 & -2.65
\end{array}\right], \quad B_{2}=\left[\begin{array}{cc}
4.45 & 2.36 \\
-1.2 & 5
\end{array}\right] \text {, } \\
& C_{0}=\left[\begin{array}{cc}
-3.32 & 1.32 \\
-1.5 & 2.93
\end{array}\right], \quad C_{1}=\left[\begin{array}{cc}
-3.05 & -1.3 \\
1.4 & 3.46
\end{array}\right] \text {, } \\
& C_{2}=\left[\begin{array}{cc}
-2.75 & -2.16 \\
0.62 & -1.75
\end{array}\right], \quad D_{0}=\left[\begin{array}{cc}
-4.05 & -2.6 \\
-2.02 & 3
\end{array}\right] \text {, } \\
& D_{1}=\left[\begin{array}{ll}
-2.12 & 2.52 \\
-1.65 & 3.89
\end{array}\right], \quad D_{2}=\left[\begin{array}{cc}
1.05 & 1.23 \\
-1.54 & 2.66
\end{array}\right] \text {, } \\
& Q_{0}=\left[\begin{array}{cc}
-1.75 & 0 \\
0 & 1.5
\end{array}\right], \quad Q_{1}=\left[\begin{array}{ll}
0 & 0 \\
0 & 1
\end{array}\right] \text {, } \\
& Q_{2}=\left[\begin{array}{cc}
2.12 & 1.52 \\
1.52 & 3
\end{array}\right], \quad \bar{Q}_{0}=\left[\begin{array}{cc}
2 & 0 \\
0 & -0.3
\end{array}\right] \text {, } \\
& \bar{Q}_{1}=\left[\begin{array}{cc}
0 & 0 \\
0 & -0.5
\end{array}\right], \bar{Q}_{2}=\left[\begin{array}{cc}
-1.12 & -0.52 \\
-0.52 & -1
\end{array}\right] \text {, } \\
& R_{0}=\left[\begin{array}{cc}
-2 & 0 \\
0 & -1
\end{array}\right], \quad R_{1}=\left[\begin{array}{ll}
1 & 0 \\
0 & 1
\end{array}\right] \text {, } \\
& R_{2}=\left[\begin{array}{cc}
1 & 0 \\
0 & 0.5
\end{array}\right], \bar{R}_{0}=\left[\begin{array}{cc}
1 & -0.5 \\
-0.5 & -1
\end{array}\right] \text {, } \\
& \bar{R}_{1}=\left[\begin{array}{cc}
-0.5 & 0 \\
0 & 1
\end{array}\right], \quad \bar{R}_{2}=\left[\begin{array}{cc}
-0.5 & 0 \\
0 & 2
\end{array}\right] \text {, } \\
& G=\left[\begin{array}{ll}
2 & 0 \\
0 & 1
\end{array}\right], \quad \bar{G}=\left[\begin{array}{cc}
-1 & 0 \\
0 & 1
\end{array}\right] \text {. }
\end{aligned}
$$

Considering the open-loop equilibrium control, from (21) and (22) (with $N=3$ ) we have

$$
\mathcal{R}_{2}+B_{2}^{T} \mathcal{P}_{3} B_{2}+D_{2}^{T} P_{3} D_{2}=\left[\begin{array}{cc}
27.7591 & -3.0114 \\
-3.0114 & 68.1710
\end{array}\right]>0
$$




$$
\begin{aligned}
& \mathcal{R}_{1}+B_{1}^{T} \mathcal{P}_{2} B_{1}+D_{1}^{T} P_{2} D_{1}=\left[\begin{array}{cc}
492.4316 & -162.4572 \\
-162.4572 & 896.5842
\end{array}\right]>0 \\
& \mathcal{R}_{0}+B_{0}^{T} \mathcal{P}_{1} B_{0}+D_{0}^{T} P_{1} D_{0}=\left[\begin{array}{cc}
10650 & 3113 \\
3113 & 13472
\end{array}\right]>0, \\
& \mathcal{W}_{2}=\left[\begin{array}{cc}
27.7591 & -3.0114 \\
-3.0114 & 68.1710
\end{array}\right] \\
& \mathcal{W}_{1}=\left[\begin{array}{cc}
473.2906 & -151.3192 \\
-140.8696 & 771.7742
\end{array}\right] \\
& \mathcal{W}_{0}=\left[\begin{array}{ll}
7133.6 & 2471.9 \\
2046.2 & 7875.8
\end{array}\right] .
\end{aligned}
$$

Note that the set of eigenvalues of $\mathcal{W}_{2}, \mathcal{W}_{1}$ and $\mathcal{W}_{0}$ are, respectively, $\{27.5359,68.3942\},\{413.7517,831.3131\}$ and $\{5.2253,9.7841\}$. Then, the corresponding (21) and (22) are solvable. Therefore, for any initial pair $t \in$ $\{0,1,2\}$ and any $x \in L_{\mathcal{F}}^{2}\left(t ; \mathbb{R}^{n}\right)$ the corresponding Problem (LQ) admits an open-loop equilibrium pair; for the initial pair $(0, x)$ with $x \in L_{\mathcal{F}}^{2}\left(0 ; \mathbb{R}^{n}\right)$, an open-loop equilibrium control is given by

$$
u^{0, x, *}=-\mathcal{W}_{k}^{\dagger} \mathcal{H}_{k} X_{k}^{0, x, *}, \quad k \in\{0,1,2\}
$$

where

$$
\begin{aligned}
\mathcal{W}_{2}^{\dagger} \mathcal{H}_{2} & =\left[\begin{array}{cc}
-0.0614 & -0.4228 \\
0.0317 & 0.0432
\end{array}\right], \\
\mathcal{W}_{1}^{\dagger} \mathcal{H}_{1} & =\left[\begin{array}{cc}
-0.0360 & -0.3667 \\
-0.2348 & -0.0076
\end{array}\right], \\
\mathcal{W}_{0}^{\dagger} \mathcal{H}_{0} & =\left[\begin{array}{cc}
0.6217 & -0.1490 \\
-0.2753 & 0.5735
\end{array}\right],
\end{aligned}
$$

and

$$
\left\{\begin{array}{l}
X_{k+1}^{0, x, *}=\left[A_{k} X_{k}^{0, x, *}+B_{k} u_{k}^{0, x, *}\right]+\left[C_{k} X_{k}^{0, x, *}+D_{k} u_{k}^{0, x, *}\right] w_{k}, \\
X_{t}^{0, x, *}=x, \quad k \in\{0,1,2\} .
\end{array}\right.
$$

On the other hand, concerned with the closed-loop equilibrium strategy, from (46) (with $N=3$ ) we have

$$
\mathcal{O}_{2}=\left[\begin{array}{cc}
27.7591 & -3.0114 \\
-3.0114 & 68.1710
\end{array}\right]>0
$$




$$
\begin{aligned}
& \mathcal{O}_{1}=\left[\begin{array}{cc}
490.5935 & -178.4499 \\
-178.4499 & 830.3166
\end{array}\right]>0, \\
& \mathcal{O}_{0}=\left[\begin{array}{ll}
6120.2 & 1693.2 \\
1693.2 & 7576.2
\end{array}\right]>0 .
\end{aligned}
$$

Therefore, the corresponding (46) is solvable, and a closed-loop equilibrium strategy is given by

$$
\begin{aligned}
\Phi_{2} & =\left[\begin{array}{cc}
0.0614 & 0.4228 \\
-0.0317 & -0.0432
\end{array}\right], \\
\Phi_{2} & =\left[\begin{array}{ll}
0.0290 & 0.3654 \\
0.2508 & 0.0124
\end{array}\right], \\
\Phi_{2} & =\left[\begin{array}{cc}
-0.6344 & 0.1815 \\
0.2718 & -0.5565
\end{array}\right] .
\end{aligned}
$$

\section{Conclusion}

In this paper, the open-loop equilibrium control and the closed-loop equilibrium strategy of a time-inconsistent mean-field stochastic LQ optimal control are investigated. Necessary and sufficient conditions are, respectively, presented for these two cases. Concerned with the existence of the openloop time-consistent equilibrium control for all the initial pairs, two coupled constrained LDEs and two coupled constrained GDREs are introduced. On the other hand, the existence of the closed-loop time-consistent equilibrium strategy is equivalent to the solvability of another two coupled constrained GDREs. Interestingly, GDREs for the open-loop formulation do not have symmetry structure, while the ones for the closed-loop formulation do have symmetric solutions. For future researches, we would like to study timeinconsistent stochastic LQ problems with other types of time-inconsistency.

\section{References}

[1] M. Ait Rami, X. Chen, and X. Y. Zhou, Discrete-time indefinite $L Q$ control with state and control dependent noises. Journal of Global Optimization, 2002, vol. 23, 245-265.

[2] S. Basak and G. Chabakauri, Dynamic mean-variance asset allocation. Rev. Financial Stud., 2010, vol. 23, 2970-3016. 
[3] T. Bjork and A. Murgoci, A general theory of Markovian time inconsisiten stochastic control problem. http://papers.ssrn.com/sol3/ papers.cfm?abstract_id=1694759, 2010.

[4] I. Ekeland and A. Lazrak, Being serious about non-commitment: subgame perfect equilibrium in continuous time. arXiv:math/0604264, 2008.

[5] I. Ekeland and T. A. Privu, Investment and consumption without commitment. Mathematics and Financial Economics, vol. 2, no. 1, pp. 57-86, 2008.

[6] Y. Hu, H. Jin, and X. Y. Zhou, Time-inconsistent stochastic linearquadratic control. SIAM Journal on Control and Optimization, 2012, vol. 50, 1548-1572.

[7] X. Li, Y. H. Ni, and J. F. Zhang, On time-consistent solution to timeinconsistent linear-quadratic optimal control of discrete-time stochastic systems. Submitted to: IEEE Transactions on Automatic Control.

[8] Y. H. Ni, X. Li, and J. F. Zhang, Indefinite mean-field stochastic linearquadratic optimal control: from finite horizon to infinite horizon. IEEE Trans. on Automatic Control, DOI : 10.1109/TAC.2015.2509958, 2016.

[9] Y. H. Ni, J. F. Zhang, and X. Li, Indefinite mean-field stochastic linearquadratic optimal control. IEEE Transactions on Automatic Control, 2015, vol. 60, no. 7, 1786-1800.

[10] R. Penrose, A generalized inverse of matrices. Mathematical Proceedings of the Cambridge Philosophical Society, 1955, vol. 52, 17-19.

[11] J. M. Yong, A deterministic linear quadratic time-inconsitent optimal control problem. Mathematical Control and Related Rields, 2011, vol. 1, no. 1, 83-118.

[12] J. M. Yong, Deterministic time-inconsistent optimal control problemsan essentially cooperative approach. Acta Appl. Math. Sinica, 2012, vol. $28,1-20$.

[13] J. M. Yong, A linear-quadratic optimal control problem for mean-field stochastic differential equations. SIAM Journal on Control Optimization, 2013, vol. 51, 2809-2838.

[14] J. M. Yong, Linear-quadratic optimal control problems for mean-field stochastic differential equations-time-consistent solutions. Electronically published by Transactions of the American Mathematical Society, DOI : 10.1090/tran/6502, 2015. 
Department of Mathematics, School of Science Tianjin Polytechnic University

Tianjin 300387, P.R. China

E-mail address: yhni@amss.ac.cn

Key Laboratory of Systems and Control

Institute of Systems Science

Academy of Mathematics and Systems Science

Chinese Academy of Sciences

BeiJing 100190, P. R. China

E-mail address: jif@iss.ac.cn 